\title{
Zircon U-Pb and Pyrite Re-Os Isotope Geochemistry of 'Skarn-Type' Fe-Cu Mineralization at the Shuikoushan Polymetallic Deposit, South China: Implications for an Early Cretaceous Mineralization Event in the Nanling Range
}

\author{
Shengbin Li ${ }^{1}{ }^{\mathbb{D}}$, Yonghua Cao ${ }^{2,3, * \mathbb{C}}$, Zeyou Song ${ }^{4,5, *}$ and Dan Xiao ${ }^{4,5}$ \\ 1 Key Laboratory of High-Temperature and High-Pressure Study of the Earth's Interior, \\ Institute of Geochemistry, Chinese Academy of Sciences, Guiyang 550081, China; lishengbin@mail.gyig.ac.cn \\ 2 Key Laboratory of Metallogenic Prediction of Nonferrous Metals and Geological Environment Monitoring, \\ Ministry of Education, School of Geosciences and Info-Physics, Central South University, \\ Changsha 410083, China \\ 3 Key Laboratory of Mineralogy and Metallogeny, Guangzhou Institute of Geochemistry, \\ Chinese Academy of Sciences, Guangzhou 510640, China \\ 4 Key Laboratory of Hunan Province for Remote Sensing Monitoring of Ecological Environment in Dongting \\ Lake Area, Center for Natural Resources Affairs of Hunan Province, Changsha 410114, China; \\ azhealuo@163.com \\ check for \\ updates \\ Citation: Li, S.; Cao, Y.; Song, Z.; \\ 5 Hunan Key Laboratory of Land Resources Evaluation and Utilization, Hunan Land and Resources Planning \\ Institute, Changsha 410007, China \\ * Correspondence: yonghuacao@gig.ac.cn (Y.C.); songzeyou@126.com (Z.S.)
} Xiao, D. Zircon U-Pb and Pyrite Re-Os Isotope Geochemistry of 'Skarn-Type' Fe-Cu Mineralization at the Shuikoushan Polymetallic Deposit, South China: Implications for an Early Cretaceous

Mineralization Event in the Nanling Range. Minerals 2021, 11, 480. https://doi.org/10.3390/min11050480

Academic Editors:

Annamaria Fornelli and

Francesca Micheletti

Received: 12 March 2021

Accepted: 27 April 2021

Published: 30 April 2021

Publisher's Note: MDPI stays neutral with regard to jurisdictional claims in published maps and institutional affiliations.

Copyright: () 2021 by the authors. Licensee MDPI, Basel, Switzerland. This article is an open access article distributed under the terms and conditions of the Creative Commons Attribution (CC BY) license (https:// creativecommons.org/licenses/by/ $4.0 /)$.

\begin{abstract}
The Shuikoushan deposit is an economic 'skarn-type' polymetallic $\mathrm{Pb}-\mathrm{Zn}$ deposit in South China. The deposit is located at the southern margin of the Hengyang basin in the northern part of the Nanling Range. Recently, economic Fe-Cu mineralization that occurs spatially connected to skarns along the contact zone between the granodiorite and limestones was discovered in the lower part of this deposit. Detailed zircon $\mathrm{U}-\mathrm{Pb}$ geochronological data indicate that the granodiorite was emplaced at $153.7 \pm 0.58 \mathrm{Ma}$ (Mean Square of Weighted Deviates $(M S W D)=2.4$ ). However, the pyrite Re-Os isochron age reveals that Fe-Cu mineralization formed at $140 \pm 11 \mathrm{Ma}(M S W D)=8.1)$, which post-dates the emplacement of the granodiorite, as well as the previously determined timing of $\mathrm{Pb}-\mathrm{Zn}$ mineralization $(157.8 \pm 1.4 \mathrm{Ma})$ in this deposit. Considering that $\mathrm{Fe}-\mathrm{Cu}$ mineralization was connected with the contact zone and also faults, and that sulfide minerals commonly occur together with quartz and calcite veins that crosscut skarns, we interpret this mineralization type as being related to injection of post-magmatic hydrothermal fluids. The timing of Fe-Cu mineralization $(140 \pm 11 \mathrm{Ma})$ is inconsistent with a long-held viewpoint that the time interval of 145 to $130 \mathrm{Ma}$ (e.g., Early Cretaceous) in the Nanling Range is a period of magmatic quiescence with insignificant mineralization, the age of 140 Ma may represent a new mineralization event in the Nanling Range.
\end{abstract}

Keywords: U-Pb zircon geochronology; zircon Lu-Hf isotopes; pyrite Re-Os isochron dating; Shuikoushan Pb-Zn deposit; Nanling Range

\section{Introduction}

The Shuikoushan $\mathrm{Pb}-\mathrm{Zn}-\mathrm{Au}$ ore field, located at the northwestern margin of the Cathaysian block (Figure 1a) and the middle part of the Nanling Range in South China, is one of the most important non-ferrous ore fields in China. The main mineral deposits in this ore field include the Shuikoushan $\mathrm{Pb}-\mathrm{Zn}$ polymetallic deposit, the Kangiiawan $\mathrm{Pb}-\mathrm{Zn}$ deposit, the Longwangshan Au deposit, and the Xianrenyan Au deposit (Figure 1b). The geological and geochemical characteristics of the ore deposits in this ore field have been investigated by numerous studies, and two general genetic models such as skarn-type 
related to contact metamorphism and hydrothermal vein-type, which resulted by infilling hydrothermal fluids into fractures have been proposed to account for their formations [1-5].



Figure 1. (a) The tectonic regimes of South China, the location of the Shuikoushan ore field is highlighted using a square. (b) Geologic map of the Shuikoushan ore field showing locations of various deposits (modified after [2,5]); the location of the Shuikoushan deposit is outlined using a blue dashed square.

The Shuikoushan deposit is an economic 'skarn-type' polymetallic $\mathrm{Pb}-\mathrm{Zn}$ deposit, with an average grade of 1.57 wt. \% Pb, $5.72 \mathrm{wt} . \% \mathrm{Zn}$, and $0.50 \mathrm{wt}$. \% Cu [6]. Previous studies conducted on this deposit have focused on geochemical characteristics and genesis of $\mathrm{Pb}-\mathrm{Zn}$ mineralization. For example, based on geological mapping and zircon $\mathrm{U}-\mathrm{Pb}$ geochronological work, $\mathrm{Pb}-\mathrm{Zn}$ mineralization in this deposit was found to be spatially related to granodiorites that have zircon U-Pb ages ranging from 153 to $163 \mathrm{Ma}$ (Table 1) [1-3,7-9]. Re-Os isochron age of the molybdenite from the Pb-Zn ore body of this deposit reveals that the timing of $\mathrm{Pb}-\mathrm{Zn}$ mineralization is $157.8 \pm 1.4 \mathrm{Ma}$, coinciding with the age of granodiorites in this deposit [9]. It is thought that $\mathrm{Pb}-\mathrm{Zn}$ mineralization and granodiorites in the Shuikoushan deposit are genetically linked [9]. Recently, the drilling program made the discovery of the No. 3 concealed granodiorite and a spatially related Fe-Cu ore body at depths from -295 to $-1050 \mathrm{~m}$ in this deposit (Figure 2). The discovery enlarges the mineral reserve and indicates a potential for prospecting other resources in the Shuikoushan deposit [4]. In addition, the Mesozoic granitoids in the Nanling Range are known to host Pb-Zn-W-Sn as the dominant mineralization types $[10,11]$, the discovery of the $\mathrm{Fe}-\mathrm{Cu}$ ore body in the Shuikoushan deposit may enrich the mineralization diversity in the Nanling Range. Albeit these significant meanings, the research on Fe-Cu mineralization in this deposit is still at a very initial stage. It is unclear whether the No. 3 granodiorite is co-genetic with other granodiorites in the Shuikoushan deposit and formed contempora- 
neously with $\mathrm{Fe}-\mathrm{Cu}$ mineralization, and what is the genesis of $\mathrm{Fe}-\mathrm{Cu}$ mineralization. In addition, the granitoid magmatism and related $\mathrm{Pb}-\mathrm{Zn}-\mathrm{W}-\mathrm{Sn}$ mineralization events in the Nanling Range were determined to have occurred mainly between 151 and 160 Ma [12-19], coeval with $\mathrm{Pb}-\mathrm{Zn}$ mineralization in the Shuikoushan deposit [9]. It is unclear whether or not Fe-Cu mineralization in this deposit is also coeval with mineralization events in the Nanling Range. Investigating this may provide implications for discovering similar types of mineralization in a much broader region. To address these problems, the emplacement age of the No. 3 granodiorite and the timing of Fe- $\mathrm{Cu}$ mineralization needs to be constrained.

Table 1. Ages of several mineralized intrusions in the Shuikoushan ore field.

\begin{tabular}{clcccc}
\hline Intrusions & Rock & Mineral & Method & Age (Ma) & Reference \\
\hline Yagongtang No. 4 & Granodiorite & Zircon & LA-ICP-MS U-Pb & $163 \pm 2$ & {$[1]$} \\
Yagongtang No. 3 & Granodiorite & Zircon & SHRIMP U-Pb & $156.33 \pm 0.64$ & {$[3]$} \\
Zhongqu & Granodiorite & Zircon & LA-ICP-MS U-Pb & $153.0 \pm 0.9$ & {$[8]$} \\
Zhongqu & Granodiorite & Zircon & LA-ICP-MS U-Pb & $156.0 \pm 1.0$ & {$[2]$} \\
Xianrenyan & Granodiorite & Zircon & LA-ICP-MS U-Pb & $156.09 \pm 0.46$ & {$[7]$} \\
Laoyachao & Granodiorite & Zircon & SIMS U-Pb & $158.8 \pm 1.8$ & {$[9]$} \\
\hline
\end{tabular}

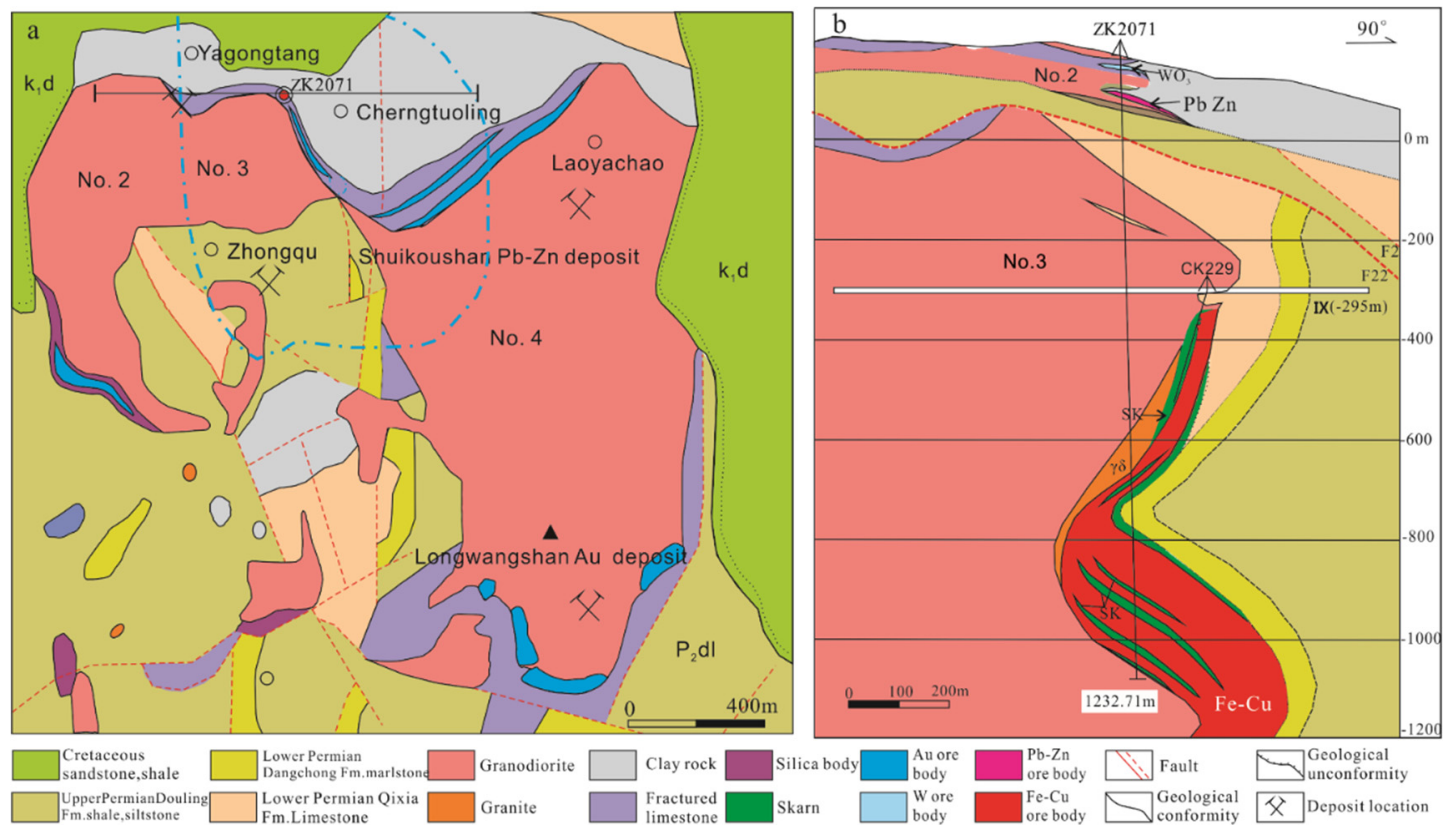

Figure 2. (a) Geologic map of the Shuikoushan deposit showing locations of different intrusions and country rocks (Modified from [20]). The concealed No. 3 granodiorite is projected on the surface (outlined by a blue dashed curve), the No. 2 and No. 4 granodioritic bodies are located at the western and eastern sides of the deposit, respectively, and are separated by a fault. (b) Cross-section of line 207 in the Shuikoushan deposit. Locations and shapes of the Pb-Zn and Fe-Cu ore bodies are displayed in this figure. The $\mathrm{Pb}-\mathrm{Zn}$ ore body is distributed mainly along the contact zone between the No. 2 granodiorite and fractured limestone, whereas the Fe-Cu ore body is distributed along the contact zone between the No. 3 granodiorite and Lower Permian Qixia limestone, note that several lenses of skarns are seen within the Fe-Cu ore body.

In this study, U-Pb zircon dating of the No. 3 concealed granodiorite was performed to constrain the emplacement age of this intrusion. Re-Os isotope system is known to be a powerful tool for dating sulfide minerals [21,22], thus the timing of Fe- $\mathrm{Cu}$ mineralization can be determined based on this method. Although the molybdenite is commonly used for Re-Os isotopic dating, it has been demonstrated that sulfide minerals with lower Re and Os concentrations such as pyrite and arsenopyrite are also suitable for Re-Os isotopic dating, and the Re-Os radiogenic system of pyrite can be used as a high-precision 
geochronometer $[23,24]$. As the Fe-Cu ore body in the Shuikoushan deposit contains abundant pyrite but lacks molybdenite, we carried out the pyrite Re-Os isochron dating to determine the timing of Fe-Cu mineralization in this study. The obtained ages are combined with whole-rock trace element compositions and trace element and Hf isotope compositions of zircons to evaluate the relationship between the No. 3 granodiorite and other granodioritic intrusions in the Shuikoushan deposit, the potential linkage between the No. 3 granodiorite and Fe-Cu mineralization, and the genesis of this mineralization type.

\section{Regional Geological Setting}

The Nanling Range in South China contains abundant Mesozoic granitoid intrusions that host significant metallic mineralization $[25,26]$. These intrusions were previously considered as S-type granitoids based on their relatively high initial $\mathrm{Sr}^{87} / \mathrm{Sr}^{86}$ ratios of $0.710-0.735$ [25,27], but were then re-identified as I- or A-type granitoids formed from highly fractionated magmas $[28,29]$. Particularly, recent studies identified several small granodioritic intrusions with relatively low initial $\mathrm{Sr}^{87} / \mathrm{Sr}^{86}$ ratios in the Range and regarded this as an I-type signature for the intrusions [9,30]. Mesozoic granitoids in Southern Hunan are hosts to numerous $\mathrm{W}-\mathrm{Sn}$ and $\mathrm{Pb}-\mathrm{Zn}$ ore deposits, which form a significant part of mineralization in the Nanling Range. Among which, W-Sn deposits are commonly related to granite plutons emplaced at 160 to $150 \mathrm{Ma}$ [15,31-33], whereas Pb-Zn deposits are usually associated with I-type granodiorite intrusions [34,35].

The Shuikoushan ore field in Southern Hunan is located in the suture zone between the Yangtze and Cathaysia blocks. The basement of this ore field consists of Precambrian metamorphic rocks that were overlain by Sinian to Mesozoic cover sedimentary successions $[2,36]$. The strata in this field are composed mainly by the Late Triassic marine carbonate and Late Triassic to Cretaceous clastic molasses Formations. Structures in the ore field consist mainly of anticline and syncline that have nearly north-south axial direction, as well as some thrust faults that strike nearly from north to south [2].

Seventy-two igneous bodies that cover a total surface area of $\sim 4.5 \mathrm{~km}^{2}$ have been discovered in this ore field. The lithology of these igneous bodies consists mainly of granodiorite and granite porphyry. The Yagongtang and Laoyachao granodiorites are two relatively large igneous bodies in the ore field, both intruded the Yagongtang inverted anticline [3]. The Yanshannian subvolcanic complex (133.9 to $199.6 \mathrm{Ma}$ ), composed mainly of dacite porphyry, andesitic tuff, rhyolite, and flow breccia, is also exposed in this ore field [37]. It is thought that $\mathrm{Pb}, \mathrm{Zn}$, and Au mineralization in this ore field is closely spatially related to the intermediate-felsic rocks of the Yanshannian subvolcanic complex [6].

\section{Ore Deposit Geology}

The Shuikoushan polymetallic deposit is located in the center of the Shuikoushan ore field (Figure 1b). The eastern part of this deposit is adjacent to the Kangiiawan $\mathrm{Pb}-\mathrm{Zn}$ deposit and the western part is adjacent to the Longwangshan Au deposit (Figure 1b). The main strata in the mining area comprise: (1) the Middle-Upper Carboniferous (Moscovian) dolomites and limestones of the Hutian Formation, (2) the Lower Permian limestone of the Qixia Formation, (3) the Lower Permian muddy, siliceous rock and marlstone of the Dangchong Formation, (4) the Upper Permian Carbonaceous shale and sandstone of the Douling Formation, (5) the Lower Cretaceous purple-red calcareous fine-grained sandstone, siltstone, and shale of the Dongjing Formation, and (6) the Quaternary sediments. Structures in this deposit include a series of nearly N-S trending folds and faults, notably the Laoyachao and Yagongtang overturned anticlines, and a large scale thrust fault named the Shiao-Kangjiawan fault (F22).

The granodioritic intrusion in this deposit was subdivided into numerous bodies by local miners, among which, No. 2, No. 3, and No. 4 are the main ones. No. 2 and No. 4 granodiorites are exposed and located at the western and eastern sides of the deposit, respectively, separated by a fault, whereas the No. 3 granodiorite is concealed and was 
discovered based on the drilling program (Figure 2a). The three rock bodies cover an area of $\sim 2.7 \mathrm{~km}^{2}$ in total and occur as lopolith and laccolith, which intruded the overturned anticline and/or fault (Figure 2a,b) [37]. The deposit contains two main mineralization types, such as $\mathrm{Pb}-\mathrm{Zn}$ and $\mathrm{Fe}-\mathrm{Cu}$. $\mathrm{Pb}-\mathrm{Zn}$ mineralization occurs dominantly along the margins of the No. 2 and No. 4 granodiorites, with minor within the (siliceous) marlstone of the Dangchong Formation. In contrast, Fe-Cu mineralization occurs along the eastern margin of the concealed No. 3 granodiorite (Figure 2b). There are also several thin lenses of $\mathrm{Au}$ mineralization found along the contact between the No. 4 granodiorite and fractured limestone (Figure 2a), details about Au mineralization can be referred to [6]. Various types of hydrothermal alteration, including chloritization, sericitization, skarnization, silicification, marbleization and hornfelization, are widespread along the contact zone between the granodiorites and country rocks. Among them, the skarnization and silicification are spatially related to $\mathrm{Pb}-\mathrm{Zn}$ and $\mathrm{Fe}-\mathrm{Cu}$ mineralization, whereas the chloritization is spatially related to Au mineralization [6].

$\mathrm{Pb}-\mathrm{Zn}$ mineralization in the Shuikoushan deposit has been described in detail by previous studies [1-3,7-9] — only a brief description is given here. Fourteen lenticular and podiform-like $\mathrm{Pb}-\mathrm{Zn}$ ore bodies were discovered, the occurrence of which is basically controlled by the contact zone between the No. 2 and No. 4 granodiorites and country rocks (e.g., the limestone of the Qixia and Huitian Formations), and include the hydrothermal vein-type, the cryptoexplosive breccia-type, and the skarn-type. Ore minerals consist mainly of galena, sphalerite, pyrite, native $\mathrm{Au}$, with small amounts of pyrrhotite, hematite, arsenopyrite, chalcopyrite, bornite, chalcocite, argentite, molybdenite, and native Ag. Gangue minerals are calcite, quartz, garnet, diopside, wollastonite, epidote, apatite, with small amounts of feldspar, fluorite, and barite. According to the exploration report [6], the average content of the $\mathrm{Pb}-\mathrm{Zn}$ ore body is $1.66 \mathrm{wt}$. \% $\mathrm{Pb}$ and $6.95 \mathrm{wt}$. \% $\mathrm{Zn}$.

Fe-Cu mineralization in the Shuikoushan deposit is spatially related to skarns that occurred along the eastern contact zone between the No. 3 granodiorite and country rocks (e.g., the limestone of the Qixia Formation and the marlstone of the Dangchong Formation) (Figures $2 \mathrm{~b}$ and $3 \mathrm{a}-\mathrm{c}$ ). The ore body of this mineralization type is buried at depth between -295 to $-1050 \mathrm{~m}$ and can be termed as a 'blind' ore body (Figure $2 \mathrm{~b}$ ). The ore body is $\sim 1200 \mathrm{~m}$ long along the strike, up to $\sim 700 \mathrm{~m}$ long toward the dipping direction, and 3.68 to $175.87 \mathrm{~m}$ ( $30.58 \mathrm{~m}$ on average) in thickness. The ore body is dominantly cylindrical, lenticular, sack-like, and stratified in shapes, but locally it can occur as veins within fissures of metamorphic rocks (Figure 3a,b). The occurrence of the ore body was controlled by the contact zone and faults and varies at different positions relative to the contact zone. For example, the ore body on the eastern side of the contact zone has a NW strike and dips to SW with a dipping angle of 55 to 70 degrees. In contrast, the 'lense-shaped' ore body located on the northern side of the contact zone has a NS or NNE strike and dips to SW with a dipping angle of 80 to 85 degrees for the upper part of the ore body and 50 to 60 degrees for the lower part of the ore body (Figure $2 \mathrm{~b}$ ). The average content is $22.2 \mathrm{wt}$ \% Fe, 0.62 wt. \% Cu, 0.3 ppm Au, and 9.35 ppm Ag [6]. Ore minerals consist mainly of magnetite, pyrite, chalcopyrite, pyrrhotite, galena, and sphalerite, with trace amounts of molybdenite and native $\mathrm{Ag}$, which are mainly anhedral to subhedral and show complex replacement and crosscutting relationships (Figure $4 \mathrm{a}-\mathrm{k}$ ). Gangue minerals are garnet, diopside, quartz, calcite, chlorite, and epidote. 

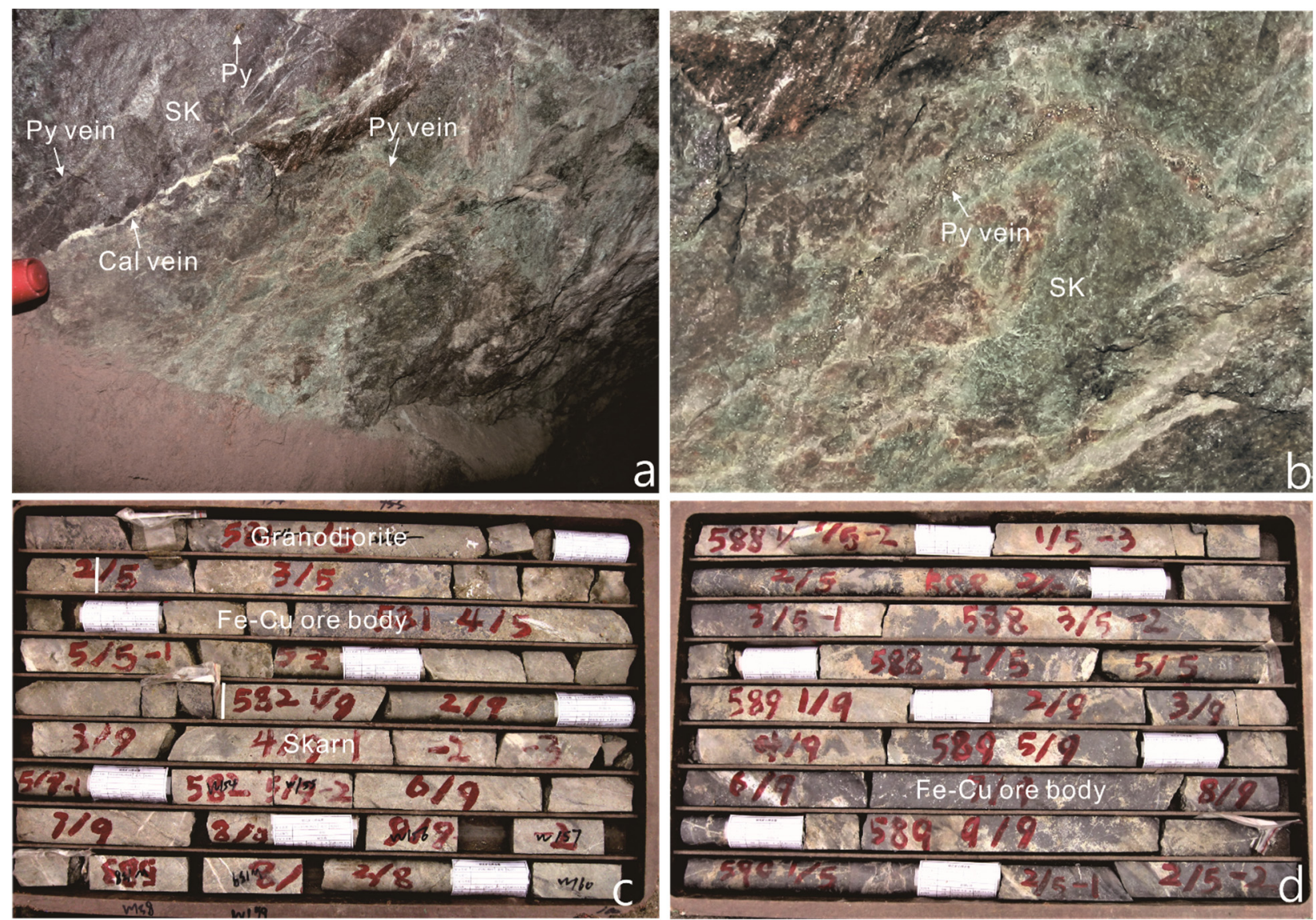

Figure 3. (a) Underground photo showing skarns at the contact zone between the No. 3 granodiorite and country rocks; (b) The close-up of photo (a) showing pyrite veinlets in the skarn. (c) Core image showing the Fe-Cu ore body at the contact zone where skarns are present, note the granodiorite rocks in the first row of the core box. (d) Core image showing numerous calcite veins (yellow-white color) occur within the Fe-Cu ore body. Abbreviations: Cal—calcite, Py-pyrite, SK-skarn. 

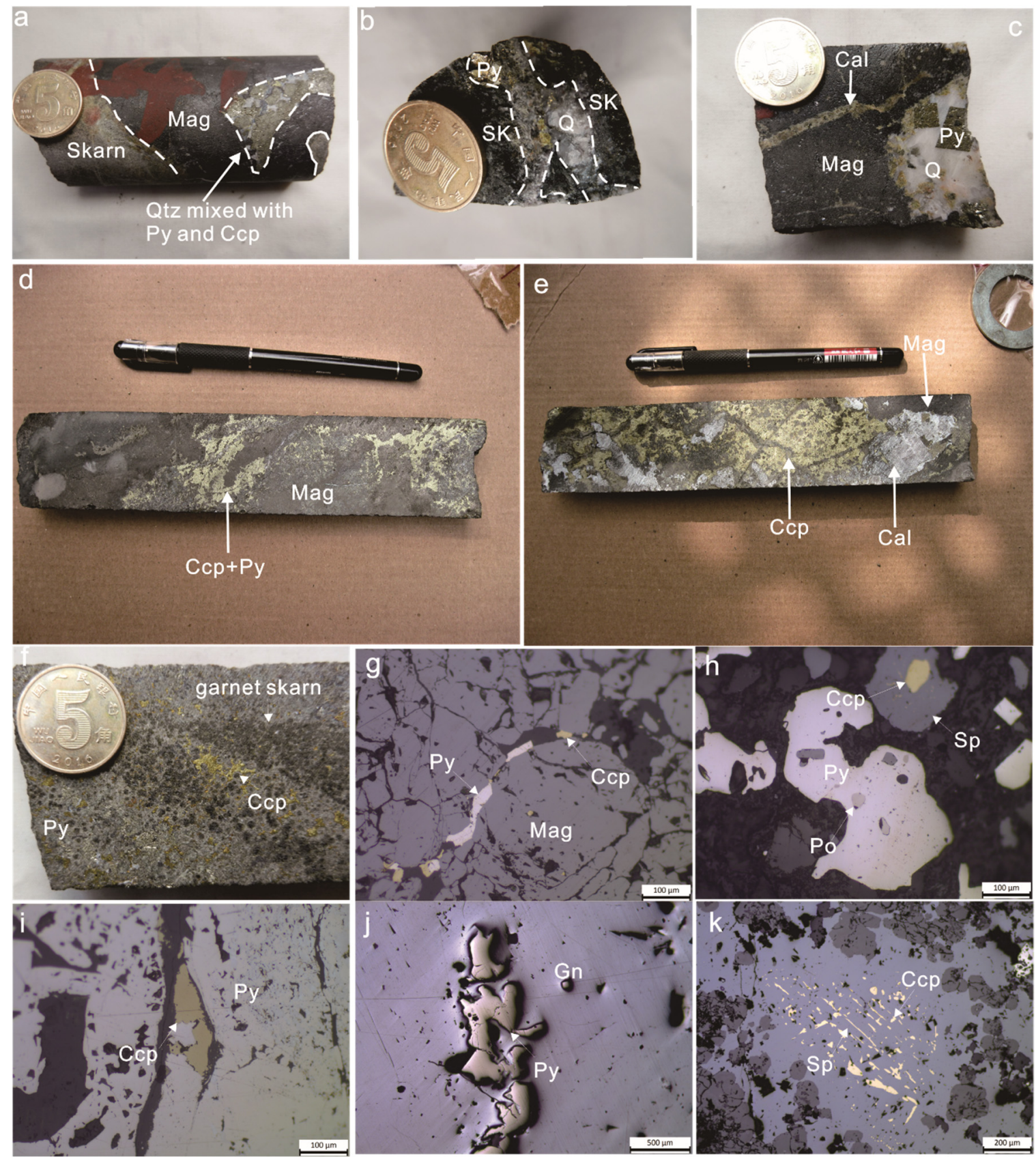

Figure 4. Typical ore hand specimens and microscopic photographs of the Shuikoushan deposit. (a) Magnetite replaced garnet skarn, pyrite-chalcopyrite-quartz veins interspersed within magnetite. (b) Pyrite-quartz vein crosscut skarns; (c) cubic pyrite grains in a quartz vein, note that a calcite vein crosscut magnetite. (d) Chalcopyrite and pyrite stringers and veinlets within the magnetite-bearing ore. (e) Massive chalcopyrite occurs together with calcite veins and blebs. (f) Disseminated to semi-massive pyrite and chalcopyrite within a garnet skarn. (g) Pyrite and chalcopyrite infilled into fissures of magnetite grains. (h) Chalcopyrite and pyrrhotite were enclosed by sphalerite and pyrite, respectively. (i) Chalcopyrite replaced pyrite along fissures of pyrite. (j) Harbor-shaped and island-like pyrite was replaced by galena. (k) Chalcopyrite was replaced by sphalerite, resulting in the 'chalcopyrite disease' texture. Abbreviations: Cal—calcite, Ccp-chalcopyrite, Gn—galena, Mag—magnetite, Qtz—quartz, Po—pyrrhotite, Py—pyrite, Sp—sphalerite.

\section{Paragenetic Sequence of $\mathrm{Fe}-\mathrm{Cu}$ Mineralization}

Based on crosscutting relationships of ore and gangue minerals, as well as textures of ore minerals, paragenetic sequence of $\mathrm{Fe}-\mathrm{Cu}$ mineralization was determined, which comprises two main episodes. From the oldest to the youngest, they are the skarn episode and the quartz-sulfide episode (Figure 5). The skarn episode refers to the calcareous 
skarn that comprises anhydrous silicate minerals such as garnet and diopside, hydrous silicate minerals such as epidote and chlorite, as well as a high amount of magnetite (Figure 5). Epidote, chlorite, and magnetite commonly occur together, which either crosscuts or replaces the garnet and diopisde (Figure 4a), based on which, the skarn episode was further subdivided into the garnet-diopside skarn and magnetite stages (Figure 5). The quartz-sulfide episode is characterized by abundant sulfide minerals, which include pyrite, chalcopyrite, pyrrhotite, sphalerite, and galena. Sulfide minerals commonly occur together with hydrothermal quartz and calcite veins (Figure $4 \mathrm{~b}-\mathrm{e}$ ), and either crosscut the magnetite (Figure $4 \mathrm{~b}-\mathrm{d}, \mathrm{g}$ ) or replaced the garnet-diopside skarn (Figure $4 \mathrm{f}$ ). This affirms that the quartz-sulfide episode occurred later than the skarn episode. Textures such as the replacement of massive pyrite by galena and sphalerite, chalcopyrite inclusion in sphalerite, and the replacement of chalcopyrite by sphalerite that resulted in the 'chalcopyrite disease' texture (Figure $4 \mathrm{~h}-\mathrm{k}$ ) are all evidence that the galena-sphalerite assemblage formed later than the pyrite-chalcopyrite assemblage, and thus the quartz-sulfide episode was further subdivided into the pyrite-chalcopyrite and galena-sphalerite stages. The pyritechalcopyrite stage comprises pyrite, chalcopyrite and pyrrhotite. The galena-sphalerite stage comprises galena, sphalerite, and minor pyrite, as well as some gangue minerals such as quartz and a small amount of carbonates.

\begin{tabular}{|c|c|c|c|c|}
\hline \multirow{2}{*}{ Mineral } & \multicolumn{2}{|c|}{ Skarn episode } & \multicolumn{2}{|c|}{ Quartz-sulfide episode } \\
\hline & $\begin{array}{l}\text { Garnet-diopside } \\
\text { stage }\end{array}$ & $\begin{array}{l}\text { Magnetite } \\
\text { stage }\end{array}$ & \begin{tabular}{|l|}
$\begin{array}{l}\text { Pyrite-chalcopyrite } \\
\text { sulfide stage }\end{array}$ \\
\end{tabular} & $\begin{array}{l}\text { Galena-Sphalerite } \\
\text { sulfide stage }\end{array}$ \\
\hline \multicolumn{5}{|l|}{ Garnet } \\
\hline \multicolumn{5}{|l|}{ Diopside } \\
\hline \multicolumn{5}{|l|}{ Epidote } \\
\hline \multicolumn{5}{|l|}{ Chlorite } \\
\hline \multicolumn{5}{|l|}{ Magnetite } \\
\hline \multicolumn{5}{|c|}{ Molybdenite } \\
\hline \multicolumn{5}{|c|}{\begin{tabular}{l|l} 
Pyrite \\
\end{tabular}} \\
\hline \multicolumn{5}{|l|}{ Quartz } \\
\hline \multicolumn{5}{|c|}{ Chalcopyrite } \\
\hline \multicolumn{5}{|l|}{ Pyrrhotite } \\
\hline \multicolumn{5}{|l|}{ Sphalerite } \\
\hline \multicolumn{5}{|l|}{ Galena } \\
\hline Calcite & & & & -- \\
\hline
\end{tabular}

Figure 5. Paragenetic sequence of ore and gangue minerals in the Fe-Cu ore body of the Shuikoushan deposit.

\section{Sampling and Analytical Methods}

\subsection{Sample Collection}

Ten samples of the No. 3 granodiorite were collected. The samples are medium- to coarse-grained and show porphyritic-like texture. They comprise a decreasing amount 
of plagioclase (22 to 28 modal\%), orthoclase (19 to 23 modal\%), quartz (20 to 30 modal\%), hornblende (10 to 18 modal $\%$ ), and biotite ( 8 to 18 modal $\%$ ), with minor amounts of apatite and zircon. Each of the samples was cut into three parts; one part was pulverized into powders for whole-rock analysis, another part was made into polished thin sections for petrography, the last part was crushed and prepared for hand-picking zircon separates. Five pieces of Fe-Cu ores were also sampled in order to obtain pyrite separates for Re-Os isotopic analysis. Locations of the collected Fe-Cu ores are given in Table 2.

Table 2. Sampling locations of pyrite separates selected for Re-Os isochron dating, collected from the Fe-Cu ore body.

\begin{tabular}{ccc}
\hline Sample No. & Sample Descriptions & Sample Locations \\
\hline ZK2071-1 & Massive pyrite-magnetite ore & Drill hole ZK2071-1@ 997.82 m \\
ZK2071-7 & Garnet skarn that contains disseminated chalcopyrite and pyrite & Drill hole ZK2071-1@ 1094.8 m \\
ZK2071-8 & Massive chalcopyrite-pyrite-magnetite ore & Drill hole ZK2071-1@ 1089.9 m \\
ZK2071-10 & Massive pyrite-magnetite ore & Drill hole ZK2071-1@ 1070 m \\
ZK2071-11 & Magnetite ore that contains pyrite-quartz veins & Drill hole ZK2071-1@ 1199 m \\
\hline
\end{tabular}

\subsection{Analytical Methods}

\subsubsection{Whole-Rock Major and Trace Element Analysis}

Powdered samples of the No. 3 granodiorite were sent to ALS Minerals-Chemex (Guangzhou) Co., Ltd. for whole-rock analysis, using an Agilent $7700 \times$ inductively coupled plasma-mass spectrometer (ICP-MS). The powdered samples $(\sim 2 \mathrm{~g})$ were mixed homogeneously with lithium borate and placed in a furnace at $1000{ }^{\circ} \mathrm{C}$ for melting. After that, the melt was cooled, dissolved with $100 \mathrm{~mL}$ of $4 \%$ nitric acid solution, and then analyzed by ICP-MS. Quality control was conducted based on ALS Chemex quality-control procedures that involve analyses on a wide array of standards, blanks, and duplicates (e.g., GBM 306-12, GBM 315-13, NCSDC 73303, OREAS 195) that were included with each batch of samples. The standard deviation of the standard is better than $3 \%$. The precision is better than $5 \%$ for most elements. The obtained whole-rock major and trace element analytical results are given in Table 3.

Table 3. Whole-rock major oxide (wt.\%) and trace element (ppm) compositions of rocks from the No. 3 granodiorite.

\begin{tabular}{|c|c|c|c|c|c|c|c|c|c|c|}
\hline $\begin{array}{c}\text { Sample } \\
\text { No. }\end{array}$ & ZK2001-1 & ZK2001-2 & ZK2001-3 & ZK2001-4 & ZK2001-5 & ZK2001-6 & ZK2001-7 & ZK2001-8 & ZK2001-9 & ZK2001-10 \\
\hline $\mathrm{SiO}_{2}$ & 57.41 & 56.57 & 56.62 & 62.29 & 55.78 & 56.23 & 60.83 & 60.31 & 59.83 & 61.48 \\
\hline $\mathrm{Al}_{2} \mathrm{O}_{3}$ & 16.04 & 17.61 & 16.28 & 15.72 & 15.76 & 16.98 & 15.98 & 16.01 & 15.52 & 15.86 \\
\hline TFeO & 7.62 & 7.31 & 7.67 & 6.42 & 7.82 & 7.38 & 7.07 & 7.56 & 6.3 & 6.55 \\
\hline $\mathrm{CaO}$ & 5.89 & 5.81 & 5.3 & 3.42 & 8.51 & 6.47 & 3.95 & 4.2 & 4.36 & 4.1 \\
\hline $\mathrm{MgO}$ & 3.28 & 2.91 & 3.53 & 2.37 & 3.85 & 3.51 & 2.48 & 2.47 & 2.49 & 2.44 \\
\hline $\mathrm{Na}_{2} \mathrm{O}$ & 2.91 & 3.04 & 2.95 & 2.85 & 3.43 & 2.93 & 2.96 & 2.89 & 3.07 & 2.95 \\
\hline $\mathrm{K}_{2} \mathrm{O}$ & 3.69 & 4.04 & 3.63 & 4.06 & 2.42 & 3.91 & 3.71 & 3.53 & 4.24 & 3.87 \\
\hline $\mathrm{Cr}_{2} \mathrm{O}_{3}$ & 0.01 & 0.02 & 0.01 & 0.01 & 0.01 & 0.01 & 0.01 & 0.01 & 0.01 & 0.01 \\
\hline $\mathrm{TiO}_{2}$ & 0.95 & 1.13 & 1.14 & 0.64 & 0.53 & 1.12 & 0.68 & 0.71 & 0.66 & 0.67 \\
\hline $\mathrm{MnO}$ & 0.13 & 0.12 & 0.12 & 0.12 & 0.19 & 0.15 & 0.13 & 0.16 & 0.15 & 0.14 \\
\hline $\mathrm{P}_{2} \mathrm{O}_{5}$ & 0.41 & 0.38 & 0.44 & 0.33 & 0.41 & 0.41 & 0.35 & 0.41 & 0.34 & 0.36 \\
\hline $\mathrm{SrO}$ & 0.08 & 0.09 & 0.07 & 0.06 & 0.08 & 0.09 & 0.07 & 0.06 & 0.06 & 0.07 \\
\hline $\mathrm{BaO}$ & 0.1 & 0.11 & 0.1 & 0.08 & 0.05 & 0.11 & 0.08 & 0.07 & 0.09 & 0.1 \\
\hline $\mathrm{LOI}^{*}$ & 0.99 & 0.79 & 2.28 & 1.36 & 1.09 & 1.08 & 1.33 & 1.87 & 2.77 & 1.16 \\
\hline Total & 99.51 & 99.93 & 100.14 & 99.73 & 99.93 & 100.38 & 99.63 & 100.26 & 99.89 & 99.76 \\
\hline $\mathrm{A} / \mathrm{CNK}^{* *}$ & 0.82 & 0.88 & 0.88 & 1.03 & 0.66 & 0.81 & 0.99 & 0.99 & 0.88 & 0.96 \\
\hline $\mathrm{A} / \mathrm{NK}^{* * *}$ & 1.82 & 1.88 & 1.85 & 1.73 & 1.91 & 1.87 & 1.80 & 1.86 & 1.61 & 1.75 \\
\hline $\mathrm{Rb}$ & 126.5 & 145.5 & 128.5 & 149 & 86.3 & 144 & 140.5 & 137.5 & 152 & 134 \\
\hline $\mathrm{Sr}$ & 717 & 834 & 603 & 602 & 708 & 794 & 636 & 625 & 534 & 661 \\
\hline Y & 22.9 & 20.6 & 20.9 & 22.7 & 32.8 & 22.6 & 24.2 & 27.8 & 24 & 23.3 \\
\hline $\mathrm{Zr}$ & 189 & 210 & 232 & 165 & 131 & 197 & 178 & 177 & 177 & 173 \\
\hline $\mathrm{Nb}$ & 9.8 & 10.6 & 12.7 & 12.8 & 5.8 & 10.3 & 13.7 & 14.8 & 13.3 & 13.3 \\
\hline $\mathrm{Ba}$ & 896 & 954 & 795 & 755 & 423 & 952 & 743 & 671 & 750 & 832 \\
\hline $\mathrm{La}$ & 37.2 & 39.1 & 40.3 & 44.9 & 41.8 & 39.8 & 44.2 & 50 & 40.4 & 40.5 \\
\hline $\mathrm{Ce}$ & 74.2 & 76.4 & 79.1 & 85.4 & 89.7 & 78.7 & 85.6 & 98.9 & 81.8 & 80.6 \\
\hline $\operatorname{Pr}$ & 8.6 & 8.8 & 8.83 & 9.46 & 10.7 & 9.09 & 9.7 & 11.3 & 9.69 & 9.29 \\
\hline $\mathrm{Nd}$ & 33.1 & 33.8 & 34.7 & 36.3 & 43.3 & 35.7 & 37.9 & 44 & 37.8 & 36.4 \\
\hline $\mathrm{Sm}$ & 6.15 & 6.41 & 6.2 & 7.29 & 8.69 & 7.08 & 7.52 & 8.61 & 7.61 & 7.12 \\
\hline $\mathrm{Eu}$ & 1.68 & 1.8 & 1.6 & 1.63 & 2.26 & 1.75 & 1.7 & 1.93 & 1.7 & 1.72 \\
\hline $\mathrm{Gd}$ & 5.14 & 4.87 & 5.19 & 5.22 & 6.77 & 5.26 & 5.69 & 6.95 & 5.62 & 5.54 \\
\hline $\mathrm{Tb}$ & 0.76 & 0.69 & 0.71 & 0.8 & 1.05 & 0.76 & 0.82 & 0.98 & 0.81 & 0.77 \\
\hline Dy & 4.25 & 3.86 & 4.04 & 4.26 & 6.16 & 4.42 & 4.53 & 5.36 & 4.51 & 4.58 \\
\hline
\end{tabular}


Table 3. Cont

\begin{tabular}{|c|c|c|c|c|c|c|c|c|c|c|}
\hline $\begin{array}{c}\text { Sample } \\
\text { No. }\end{array}$ & ZK2001-1 & ZK2001-2 & ZK2001-3 & ZK2001-4 & ZK2001-5 & ZK2001-6 & ZK2001-7 & ZK2001-8 & ZK2001-9 & ZK2001-10 \\
\hline Ho & 0.85 & 0.77 & 0.75 & 0.86 & 1.26 & 0.86 & 0.89 & 1.04 & 0.93 & 0.92 \\
\hline Er & 2.49 & 2.08 & 2.09 & 2.28 & 3.52 & 2.42 & 2.46 & 2.89 & 2.48 & 2.4 \\
\hline Tm & 0.37 & 0.31 & 0.31 & 0.32 & 0.52 & 0.36 & 0.36 & 0.43 & 0.37 & 0.36 \\
\hline $\mathrm{Yb}$ & 2.31 & 2.04 & 2.08 & 2.07 & 3.45 & 2.29 & 2.38 & 2.65 & 2.42 & 2.32 \\
\hline $\mathrm{Lu}$ & 0.36 & 0.31 & 0.32 & 0.34 & 0.52 & 0.35 & 0.36 & 0.39 & 0.34 & 0.34 \\
\hline $\mathrm{Hf}$ & 5.8 & 6 & 6.7 & 4.8 & 4.1 & 5.8 & 5.6 & 5.3 & 5.5 & 5.4 \\
\hline $\mathrm{Ta}$ & 0.7 & 0.8 & 0.9 & 0.9 & 0.9 & 0.8 & 1.1 & 1.1 & 1 & 1 \\
\hline Th & 10.5 & 10.25 & 10.75 & 12.25 & 7.29 & 9.39 & 12.5 & 13.05 & 13.8 & 13.35 \\
\hline $\mathrm{Y}$ & 22.9 & 20.6 & 20.9 & 22.7 & 32.8 & 22.6 & 24.2 & 27.8 & 24 & 23.3 \\
\hline$\sum$ LREE $^{* * * *}$ & 160.93 & 166.31 & 170.73 & 184.98 & 196.45 & 172.12 & 186.62 & 214.74 & 179 & 175.63 \\
\hline$\sum_{* * * * *} \mathrm{HREE}$ & 39.43 & 35.53 & 36.39 & 38.85 & 56.05 & 39.32 & 41.69 & 48.49 & 41.48 & 40.53 \\
\hline$\sum_{* * * * * *} \mathrm{REE}$ & 200.36 & 201.84 & 207.12 & 223.83 & 252.5 & 211.44 & 228.31 & 263.23 & 220.48 & 216.16 \\
\hline $\mathrm{Eu} / \mathrm{Eu}^{*}$ & 0.91 & 0.98 & 0.86 & 0.81 & 0.90 & 0.88 & 0.79 & 0.76 & 0.79 & 0.84 \\
\hline $\mathrm{Ce} / \mathrm{Ce}^{*}$ & 1.15 & 1.14 & 1.15 & 1.15 & 1.15 & 1.13 & 1.14 & 1.15 & 1.14 & $\begin{array}{l}0.14 \\
1.14\end{array}$ \\
\hline
\end{tabular}

${ }^{*}$ Loss on ignition; ${ }^{* *} \mathrm{~A} / \mathrm{CNK}=\mathrm{Al}_{2} \mathrm{O}_{3} /\left(\mathrm{CaO}+\mathrm{Na}_{2} \mathrm{O}+\mathrm{K}_{2} \mathrm{O}\right) ;{ }^{* * *} \mathrm{~A} / \mathrm{NK}=\mathrm{Al}_{2} \mathrm{O}_{3} /\left(\mathrm{Na}_{2} \mathrm{O}+\mathrm{K}_{2} \mathrm{O}\right) ;{ }^{* * *}$ Total abundances of light rare earth elements; ${ }^{* * * * *}$ Total abundances of heavy rare earth elements; ${ }^{* * * * *}$ Total abundances of rare earth elements; $\mathrm{Eu} / \mathrm{Eu}^{*}=(\mathrm{Eu})_{\mathrm{N}} /\left[(\mathrm{Sm})_{\mathrm{N}} \times\right.$ $\left.(\mathrm{Gd})_{\mathrm{N}}\right]^{0.5}, \mathrm{Ce} / \mathrm{Ce} \mathrm{e}^{*}=(\mathrm{Ce})_{\mathrm{N}} /\left[(\mathrm{La})_{\mathrm{N}} \times(\mathrm{Nd})_{\mathrm{N}}\right]^{0.5}$.

\subsubsection{U-Pb Dating and Trace Elements in Zircon}

Based on petrographic observations, zircon grains were sorted using a combination of heavy liquid and magnetic separation techniques. Transparent, crack- and inclusion-free zircon grains with good crystal shapes were hand-picked and mounted in epoxy resin. After the resin was fully cured, the epoxy disc was polished until the zircon was exposed. Cathodoluminescence (CL) and back-scattered electron (BSE) images of zircon grains were obtained using a JEOL-JXA-8100 electron probe micro-analyzer at China Metallurgical Geology Bureau, aiming to characterize the internal textures of zircon grains and identify suitable targets for spot analyses. The operating conditions include an acceleration voltage of $15 \mathrm{kV}$ and a beam current of $2 \times 10^{-8} \mathrm{~A}$. Nineteen zircon grains that are 100 to $180 \mu \mathrm{m}$ long and 60 to $120 \mu \mathrm{m}$ wide with an aspect ratio of 1.6 to 3.0 were analyzed. The CL images show that the analyzed zircons have typical oscillatory zoning textures (Figure 6).
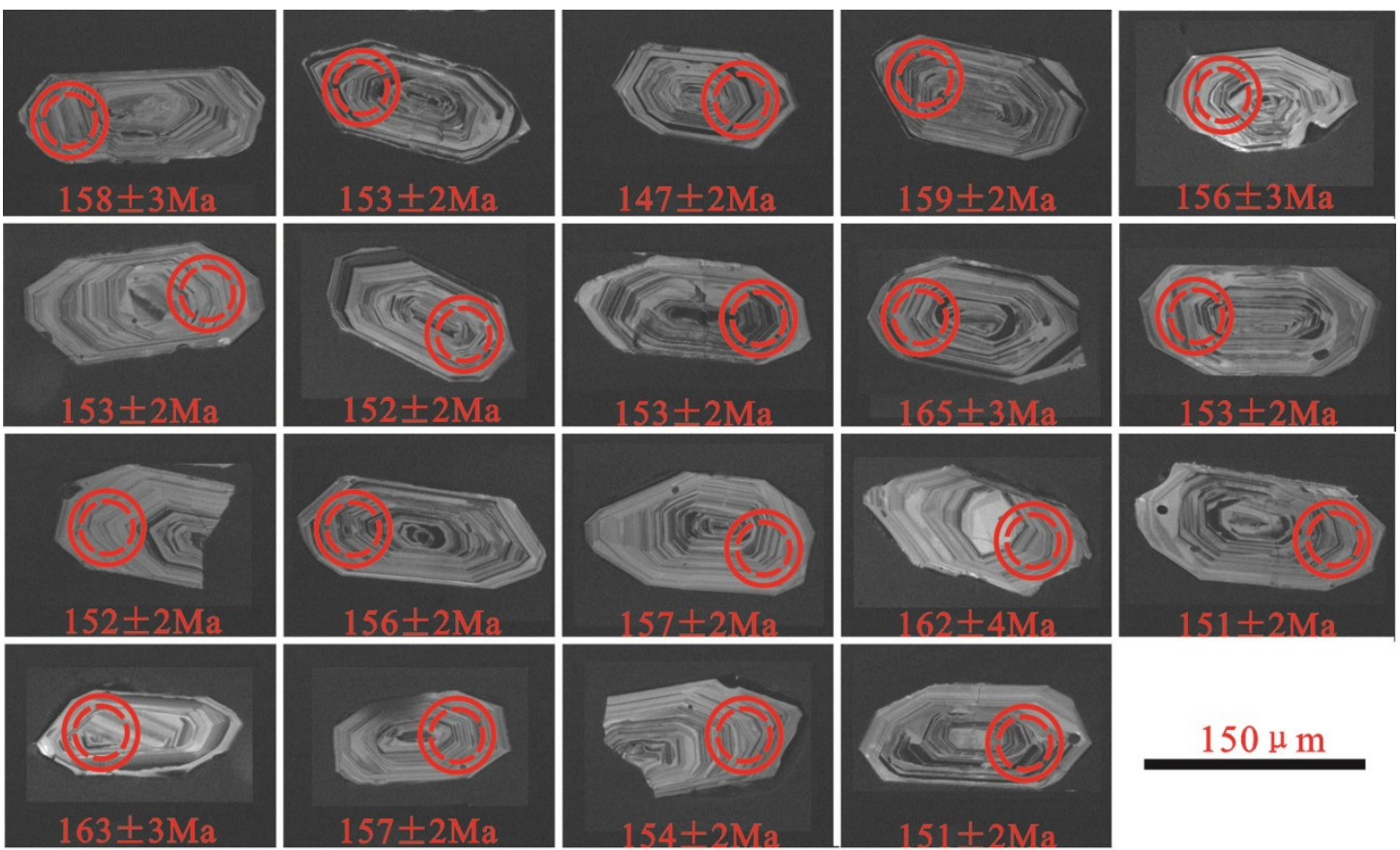

\section{$150 \mu \mathrm{m}$}

Figure 6. Cathodoluminescence images of zircon grains collected from the No. 3 granodiorite in the Shuikoushan deposit. The dashed red circles represent laser pits ( $\sim 30 \mathrm{um})$ for zircon trace element and U-Pb isotopic analyses, the solid red circles represent laser pits for zircon $\mathrm{Hf}$ isotopic analyses ( $50 \mathrm{um})$. 
Trace element compositions and $\mathrm{U}-\mathrm{Pb}$ isotope ratios of zircons were acquired using a laser ablation inductively coupled-plasma spectrometry (LA-ICP-MS) at China Metallurgical Geology Bureau. The Thermo Scientific XSeries 2 ICP-MS instrument was coupled to a Compex Pro $193 \mathrm{~nm}$ ArF excimer laser ablation system. The ablation pits are $\sim 30 \mu \mathrm{m}$ in diameter and $\sim 40 \mu \mathrm{m}$ in depth. The density of the laser energy was $8.5 \mathrm{~J} / \mathrm{cm}^{2}$ and ablation frequency was $8 \mathrm{~Hz}$. Helium gas was used as a carrier gas. Multiple spots per grain were analyzed to test both grain-to-grain and intra-grain variability. Standards were analyzed throughout the sequence to allow for drift correction. A NIST 610 silicate glass standard was used as a monitor standard for trace elements to assess the accuracy and precision of the runs. ${ }^{90} \mathrm{Zr}$ was used as the internal standard assuming stoichiometric values for zircon, in order to calibrate the trace element concentrations of zircons. The obtained trace element compositions of zircons are tabulated in Table 4. The standard GJ-1 zircon (599.8 $\pm 4.5 \mathrm{Ma}$; [38]) was used for calibration of the acquired zircon U-Pb isotope ratios. The standard Plesovice zircon (337.13 $\pm 0.37 \mathrm{Ma}$; [39]) was treated as an unknown sample during analysis, in order to check the accuracy of the acquired zircon $\mathrm{U}-\mathrm{Pb}$ isotope ratios. Off-line data reduction was carried out using the ICPMSDataCal software (version 11, the State Key Laboratory of Geological Processes and Mineral Resources, China University of Geosciences, Wuhan, China) [40]. Concordia diagram and weighted mean calculations were performed using the ISOPLOT software [41]. The obtained zircon U-Pb geochronology results are tabulated in Table 5.

Table 4. The Ti and rare earth element (REE) contents (ppm) of zircons from the No. 3 granodiorite of the Shuikoushan deposit.

\begin{tabular}{|c|c|c|c|c|c|c|c|c|c|c|c|c|c|c|c|}
\hline No. & $\mathbf{T i}$ & La & $\mathrm{Ce}$ & $\operatorname{Pr}$ & $\mathrm{Nd}$ & Sm & $\mathbf{E u}$ & $\mathrm{Gd}$ & $\mathrm{Tb}$ & Dy & Ho & Er & $\operatorname{Tm}$ & $\mathrm{Yb}$ & $\mathbf{L u}$ \\
\hline 01 & 3.228 & 0.435 & 24.556 & 0.131 & 1.099 & 2.132 & 0.888 & 12.121 & 4.345 & 56.862 & 23.603 & 118.003 & 28.113 & 293.487 & 65.303 \\
\hline 02 & 5.482 & 0.120 & 13.058 & 0.029 & 0.535 & 1.284 & 0.771 & 9.983 & 4.093 & 58.119 & 25.590 & 134.226 & 33.215 & 348.903 & 78.947 \\
\hline 03 & 3.114 & 0.047 & 30.350 & 0.069 & 0.981 & 2.110 & 0.935 & 13.597 & 4.603 & 57.778 & 23.340 & 113.556 & 27.376 & 285.894 & 63.469 \\
\hline 04 & 3.474 & 0.002 & 16.368 & 0.043 & 0.659 & 2.041 & 0.960 & 14.704 & 5.646 & 76.613 & 32.702 & 168.069 & 40.053 & 425.247 & 93.043 \\
\hline 05 & 2.860 & 0.130 & 21.395 & 0.084 & 1.097 & 1.811 & 0.842 & 10.957 & 4.012 & 53.452 & 22.320 & 114.337 & 27.836 & 296.059 & 67.230 \\
\hline 06 & 2.983 & 11.461 & 41.310 & 3.062 & 14.161 & 3.774 & 0.901 & 9.836 & 3.113 & 37.137 & 15.103 & 75.570 & 18.219 & 195.684 & 43.690 \\
\hline 07 & 3.848 & 0.516 & 27.970 & 0.205 & 1.540 & 2.154 & 1.129 & 15.351 & 5.916 & 79.187 & 33.152 & 170.919 & 40.363 & 423.611 & 93.754 \\
\hline 08 & 1.576 & 0.018 & 22.718 & 0.041 & 0.671 & 2.114 & 1.266 & 17.297 & 6.730 & 93.315 & 38.839 & 198.954 & 46.658 & 482.166 & 103.850 \\
\hline 09 & 3.197 & 1.971 & 19.169 & 0.578 & 2.465 & 1.335 & 0.538 & 7.533 & 2.697 & 38.545 & 16.047 & 83.095 & 20.693 & 225.309 & 49.877 \\
\hline 10 & 45.200 & 33.614 & 94.123 & 8.816 & 37.429 & 8.301 & 1.710 & 16.953 & 4.759 & 57.484 & 22.899 & 114.241 & 26.618 & 281.604 & 62.346 \\
\hline 11 & 3.994 & 0.404 & 19.713 & 0.135 & 0.959 & 1.676 & 0.793 & 10.890 & 4.259 & 54.364 & 22.683 & 115.100 & 27.815 & 297.425 & 65.906 \\
\hline 12 & 4.927 & 0.424 & 20.988 & 0.160 & 1.254 & 1.635 & 0.768 & 10.380 & 3.750 & 48.152 & 20.093 & 99.709 & 24.157 & 256.117 & 56.331 \\
\hline 13 & 0.966 & 5.778 & 29.464 & 1.367 & 6.075 & 2.506 & 0.724 & 8.894 & 3.265 & 39.596 & 16.176 & 81.162 & 19.866 & 209.479 & 47.256 \\
\hline 14 & 0.206 & 0.008 & 13.712 & 0.019 & 0.377 & 0.806 & 0.466 & 6.418 & 2.433 & 30.203 & 12.970 & 68.028 & 16.608 & 180.337 & 41.119 \\
\hline 15 & 0.660 & 0.029 & 20.154 & 0.048 & 0.722 & 1.654 & 0.803 & 11.111 & 4.045 & 52.367 & 22.040 & 112.704 & 27.207 & 286.627 & 63.530 \\
\hline 16 & 2.931 & 1.131 & 24.220 & 0.490 & 3.082 & 2.868 & 1.303 & 17.629 & 6.620 & 86.960 & 35.724 & 177.957 & 41.700 & 422.506 & 90.524 \\
\hline 17 & 2.210 & 0.001 & 16.142 & 0.035 & 0.555 & 1.242 & 0.559 & 7.652 & 2.834 & 35.945 & 15.010 & 77.154 & 18.843 & 203.044 & 46.329 \\
\hline 18 & 2.438 & 0.224 & 18.914 & 0.083 & 0.937 & 1.649 & 0.676 & 8.986 & 3.266 & 41.852 & 17.777 & 90.109 & 21.785 & 235.543 & 53.203 \\
\hline 19 & 4.308 & 11.043 & 66.485 & 3.389 & 16.419 & 6.226 & 2.111 & 24.537 & 8.610 & 111.155 & 45.426 & 223.852 & 51.774 & 542.977 & 116.546 \\
\hline
\end{tabular}


Table 5. Zircon U-Pb isotopic compositions and geochronological results of the No. 3 granodiorite in the Shuikoushan deposit.

\begin{tabular}{|c|c|c|c|c|c|c|c|c|c|c|c|c|c|c|}
\hline \multirow{2}{*}{ No. } & ${ }^{204} \mathrm{~Pb}$ & $\mathrm{~Pb}$ & Th & $\mathrm{U}$ & \multirow{2}{*}{$\mathrm{Th} / \mathrm{U}$} & \multicolumn{6}{|c|}{ Isotope Ratios } & \multicolumn{3}{|c|}{ Age (Ma) } \\
\hline & & (ppm) & & & & ${ }^{206} \mathrm{~Pb} /{ }^{204} \mathrm{~Pb} \pm 1 \sigma^{*}$ & ${ }^{207} \mathrm{~Pb} /{ }^{204} \mathrm{~Pb} \pm 1 \sigma$ & ${ }^{208} \mathrm{~Pb} /{ }^{204} \mathrm{~Pb} \pm 1 \sigma$ & ${ }^{207} \mathrm{~Pb} /{ }^{206} \mathrm{~Pb} \pm 1 \sigma$ & ${ }^{207} \mathrm{~Pb} /{ }^{235} \mathrm{U} \pm 1 \sigma$ & ${ }^{206} \mathrm{~Pb} /{ }^{238} \mathrm{U} \pm 1 \sigma$ & ${ }^{206} \mathrm{~Pb} /{ }^{238} \mathrm{U} \pm 1 \sigma$ & ${ }^{207} \mathrm{~Pb} /{ }^{235} \mathrm{U} \pm 1 \sigma$ & ${ }^{207} \mathrm{~Pb} /{ }^{206} \mathrm{~Pb} \pm 1 \sigma$ \\
\hline 01 & 0.2299 & 12 & 284 & 353 & 0.80 & $162.5541 \pm 3.1165$ & $8.6963 \pm 0.2343$ & $19.8415 \pm 0.5228$ & $0.0493 \pm 0.0017$ & $0.1693 \pm 0.0059$ & $0.0248 \pm 0.0004$ & $158 \pm 3$ & $159 \pm 5$ & $161 \pm 81$ \\
\hline 02 & 0.0000 & 8 & 120 & 271 & 0.44 & - & - & - & $0.0506 \pm 0.0023$ & $0.1682 \pm 0.0077$ & $0.0240 \pm 0.0004$ & $153 \pm 2$ & $158 \pm 7$ & $233 \pm 109$ \\
\hline 04 & 0.3761 & 12 & 205 & 378 & 0.54 & $105.8367 \pm 4.5492$ & $5.8363 \pm 0.2982$ & $8.537 \pm 0.3813$ & $0.0511 \pm 0.0020$ & $0.1749 \pm 0.0066$ & $0.0249 \pm 0.0003$ & $159 \pm 2$ & $164 \pm 6$ & $256 \pm 82$ \\
\hline 05 & 0.0000 & 10 & 233 & 305 & 0.76 & - & - & - & $0.0484 \pm 0.0021$ & $0.1614 \pm 0.0070$ & $0.0245 \pm 0.0004$ & $156 \pm 3$ & $152 \pm 6$ & $120 \pm 104$ \\
\hline 06 & 0.0000 & 7 & 162 & 230 & 0.70 & - & 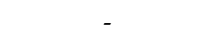 & - & $0.0476 \pm 0.0019$ & $0.1559 \pm 0.0058$ & $0.0241 \pm 0.0004$ & $153 \pm 2$ & $147 \pm 5$ & $80 \pm 96$ \\
\hline 07 & 0.4531 & 13 & 312 & 408 & 0.76 & $91.6916 \pm 4.3939$ & $4.9962 \pm 0.2996$ & $9.9324 \pm 0.6416$ & $0.0497 \pm 0.0016$ & $0.1650 \pm 0.0058$ & $0.0239 \pm 0.0003$ & $152 \pm 2$ & $155 \pm 5$ & $189 \pm 76$ \\
\hline 08 & 0.3113 & 18 & 340 & 561 & 0.61 & $186.0025 \pm 8.8115$ & $10.2026 \pm 0.5346$ & $17.5394 \pm 0.9812$ & $0.0499 \pm 0.0014$ & $0.1659 \pm 0.0047$ & $0.0241 \pm 0.0003$ & $153 \pm 2$ & $156 \pm 4$ & $191 \pm 65$ \\
\hline 09 & 1.0317 & 8 & 139 & 231 & 0.60 & $25.3724 \pm 1.5253$ & $1.5284 \pm 0.216$ & $2.3369 \pm 0.3063$ & $0.0545 \pm 0.0030$ & $0.1948 \pm 0.0109$ & $0.0260 \pm 0.0005$ & $165 \pm 3$ & $181 \pm 9$ & $391 \pm 122$ \\
\hline 11 & 0.6327 & 12 & 264 & 387 & 0.68 & $62.1847 \pm 3.2604$ & $3.3457 \pm 0.2432$ & $6.1415 \pm 0.3878$ & $0.0492 \pm 0.0017$ & $0.1626 \pm 0.0057$ & $0.0239 \pm 0.0003$ & $152 \pm 2$ & $153 \pm 5$ & $167 \pm 80$ \\
\hline 12 & 0.0000 & 10 & 244 & 313 & 0.78 & - & - & - & $0.0500 \pm 0.0018$ & $0.1709 \pm 0.0066$ & $0.0245 \pm 0.0004$ & $156 \pm 2$ & $160 \pm 6$ & $195 \pm 83$ \\
\hline 13 & 0.0000 & 9 & 216 & 278 & 0.78 & - & - & - & $0.0504 \pm 0.0018$ & $0.1725 \pm 0.0064$ & $0.0246 \pm 0.0004$ & $157 \pm 2$ & $162 \pm 6$ & $217 \pm 85$ \\
\hline 14 & 0.0000 & 6 & 108 & 195 & 0.55 & - & 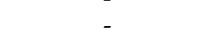 & - & $0.0553 \pm 0.0038$ & $0.1925 \pm 0.0116$ & $0.0255 \pm 0.0006$ & $162 \pm 4$ & $179 \pm 10$ & $433 \pm 152$ \\
\hline 15 & 0.7167 & 10 & 241 & 335 & 0.72 & $46.9730 \pm 2.6036$ & $2.6454 \pm 0.2306$ & $5.0501 \pm 0.3424$ & $0.0503 \pm 0.0016$ & $0.1627 \pm 0.0048$ & $0.0237 \pm 0.0004$ & $151 \pm 2$ & $153 \pm 4$ & $206 \pm 74$ \\
\hline 16 & 0.8753 & 12 & 302 & 379 & 0.80 & $44.8390 \pm 6.9567$ & $2.3401 \pm 0.3906$ & $5.0659 \pm 1.0803$ & $0.0478 \pm 0.0023$ & $0.1685 \pm 0.0079$ & $0.0256 \pm 0.0004$ & $163 \pm 3$ & $158 \pm 7$ & $100 \pm 98$ \\
\hline 17 & 0.0000 & 8 & 172 & 258 & 0.67 & - & - & - & $0.0507 \pm 0.0019$ & $0.1732 \pm 0.0063$ & $0.0247 \pm 0.0004$ & $157 \pm 2$ & $162 \pm 5$ & $228 \pm 85$ \\
\hline 18 & 0.6845 & 9 & 202 & 274 & 0.74 & $41.9192 \pm 2.0682$ & $2.3646 \pm 0.176$ & $4.4998 \pm 0.2917$ & $0.0509 \pm 0.0019$ & $0.1705 \pm 0.0064$ & $0.0242 \pm 0.0003$ & $154 \pm 2$ & $160 \pm 6$ & $235 \pm 85$ \\
\hline 19 & 0.3204 & 20 & 567 & 614 & 0.92 & $194.2122 \pm 5.4401$ & $10.5787 \pm 0.3844$ & $25.6603 \pm 0.913$ & $0.0490 \pm 0.0012$ & $0.1611 \pm 0.0105$ & $0.0237 \pm 0.0003$ & $151 \pm 2$ & $152 \pm 4$ & $150 \pm 53$ \\
\hline
\end{tabular}

* $1 \sigma$ standard derivation. Blank cells are marked with '-'. 


\subsubsection{Zircon Lu-Hf Isotopes}

In situ zircon Lu-Hf isotope analysis was performed using a Nu Plasma IImulticollector inductively coupled plasma-mass spectrometer (ICP-MS) equipped with a GeoLas 2005 laser ablation system (MC-ICPMS) at Nanjing FocuMS Technology Co., Ltd. (Nanjing, China). Analyses were performed on the pits ablated for previous U-Pb isotope analysis. The ablation pits are $\sim 50 \mu \mathrm{m}$ in diameter and $\sim 40 \mu \mathrm{m}$ in depth, with ablation time of 40 $\mathrm{s}$ and repetition rate of $8 \mathrm{~Hz}$. Five standard zircons (GJ-1, 91500, Plešovice, Mud Tank, Penglai) were analyzed between every 10 samples to monitor the reproducibility of the zircon Hf isotopic data. The average ${ }^{176} \mathrm{Lu} /{ }^{177} \mathrm{Hf}$ for GJ-1 is $0.282008 \pm 0.000025$ ( $2 \sigma$ standard deviation (SD)), for 91,500 is $0.282307 \pm 0.000031$, for Plešovice is $0.282482 \pm 0.000013$, for Mud Tank is $0.282504 \pm 0.000044$, and for Penglai is $0.282906 \pm 0.000010[39,42-44]$. Raw count rates for ${ }^{172} \mathrm{Yb},{ }^{173} \mathrm{Yb},{ }^{175} \mathrm{Lu},{ }^{176}(\mathrm{Hf}+\mathrm{Yb}+\mathrm{Lu}),{ }^{177} \mathrm{Hf},{ }^{178} \mathrm{Hf},{ }^{179} \mathrm{Hf},{ }^{180} \mathrm{Hf}$, and ${ }^{182} \mathrm{~W}$ were measured, and isobaric interference corrections for ${ }^{176} \mathrm{Lu}$ and ${ }^{176} \mathrm{Yb}$ on ${ }^{176} \mathrm{Hf}$ were made, details were given in Ref. [45]. The off-line processing of analytical data was performed using ICPMSDataCal software, including the selection of samples and blank signals and the correction of isotope mass fractionation [40]. The obtained zircon Hf isotopic compositions are tabulated in Table 6.

Table 6. The Hf isotopic compositions of zircons from No. 3 granodiorite in Shuikoushan deposit.

\begin{tabular}{|c|c|c|c|c|c|c|c|c|c|c|c|}
\hline No. & $\begin{array}{l}\text { Age } \\
\text { (Ma) }\end{array}$ & $176 \mathrm{Yb} / 177 \mathrm{Hf}$ & $2 \sigma$ & 176Lu/177Hf & $2 \sigma$ & 176Hf/177Hf & $2 \sigma$ & 178Hf/177Hf & $2 \sigma$ & $\varepsilon \mathbf{H f}(\mathbf{t})$ & $\begin{array}{c}T_{\mathrm{DM} 2} \\
\text { (Ma) }\end{array}$ \\
\hline 01 & 158 & 0.043373 & 0.000140 & 0.001518 & 0.000006 & 0.282481 & 0.000012 & 1.46793 & 0.00004 & -7.0 & 1644 \\
\hline 02 & 153 & 0.046387 & 0.000409 & 0.001656 & 0.000008 & 0.282460 & 0.000012 & 1.46776 & 0.00003 & -7.8 & 1694 \\
\hline 03 & 147 & 0.038990 & 0.000131 & 0.001389 & 0.000004 & 0.282451 & 0.000009 & 1.46786 & 0.00003 & -8.3 & 1715 \\
\hline 04 & 159 & 0.044439 & 0.000186 & 0.001545 & 0.000003 & 0.282406 & 0.000011 & 1.46785 & 0.00003 & -9.6 & 1809 \\
\hline 05 & 156 & 0.047420 & 0.000154 & 0.001629 & 0.000008 & 0.282437 & 0.000010 & 1.46782 & 0.00003 & -8.6 & 1743 \\
\hline 06 & 153 & 0.035784 & 0.000142 & 0.001210 & 0.000004 & 0.282459 & 0.000008 & 1.46779 & 0.00002 & -7.8 & 1693 \\
\hline 07 & 152 & 0.043828 & 0.000373 & 0.001560 & 0.000012 & 0.282433 & 0.000009 & 1.46785 & 0.00003 & -8.8 & 1754 \\
\hline 08 & 153 & 0.045697 & 0.000598 & 0.001607 & 0.000017 & 0.282414 & 0.000010 & 1.46780 & 0.00003 & -9.5 & 1795 \\
\hline 09 & 165 & 0.057360 & 0.000492 & 0.001714 & 0.000011 & 0.282427 & 0.000011 & 1.46781 & 0.00003 & -8.8 & 1761 \\
\hline 10 & 153 & 0.048127 & 0.000220 & 0.001528 & 0.000010 & 0.282435 & 0.000010 & 1.46779 & 0.00003 & -8.7 & 1748 \\
\hline 11 & 152 & 0.037208 & 0.000183 & 0.001202 & 0.000009 & 0.282460 & 0.000011 & 1.46784 & 0.00003 & -7.8 & 1692 \\
\hline 12 & 156 & 0.046840 & 0.000210 & 0.001515 & 0.000005 & 0.282457 & 0.000008 & 1.46776 & 0.00002 & -7.9 & 1698 \\
\hline 13 & 157 & 0.053740 & 0.000457 & 0.001555 & 0.000018 & 0.282462 & 0.000009 & 1.46778 & 0.00002 & -7.7 & 1687 \\
\hline 14 & 162 & 0.017525 & 0.000212 & 0.000627 & 0.000006 & 0.281848 & 0.000012 & 1.46785 & 0.00003 & -29.2 & 3023 \\
\hline 15 & 151 & 0.053747 & 0.000232 & 0.001729 & 0.000006 & 0.282457 & 0.000008 & 1.46781 & 0.00003 & -8.0 & 1702 \\
\hline 16 & 163 & 0.044762 & 0.000240 & 0.001482 & 0.000012 & 0.282435 & 0.000009 & 1.46777 & 0.00003 & -8.5 & 1743 \\
\hline 17 & 157 & 0.039111 & 0.000178 & 0.001440 & 0.000006 & 0.282457 & 0.000009 & 1.46779 & 0.00003 & -7.8 & 1697 \\
\hline 18 & 154 & 0.035516 & 0.000273 & 0.001108 & 0.000004 & 0.282438 & 0.000008 & 1.46782 & 0.00002 & -8.5 & 1739 \\
\hline 19 & 151 & 0.048764 & 0.000224 & 0.001539 & 0.000006 & 0.282418 & 0.000008 & 1.46777 & 0.00002 & -9.4 & 1787 \\
\hline
\end{tabular}

Note: $\varepsilon \mathrm{Hf}(\mathrm{t})=10,000 \times\left\{\left[\left({ }^{176} \mathrm{Hf} /{ }^{177} \mathrm{Hf}\right)_{S}-\left({ }^{176} \mathrm{Lu} /{ }^{177} \mathrm{Hf}\right)_{S} \times\left(\mathrm{e}^{\lambda t}-1\right)\right] /\left[\left({ }^{176} \mathrm{Hf} /{ }^{177} \mathrm{Hf}\right)_{\mathrm{CHUR} .0}-\left({ }^{176} \mathrm{Lu} /{ }^{177} \mathrm{Hf}\right)_{\mathrm{CHUR}} \times\left(\mathrm{e}^{\lambda t}-1\right)\right]-1\right\} ; T_{D M 1}$ $=1 / \lambda \times \ln \left\{1+\left[\left({ }^{176} \mathrm{Hf} /{ }^{177} \mathrm{Hf}\right)_{\mathrm{S}}-\left({ }^{176} \mathrm{Hf} /{ }^{177} \mathrm{Hf}\right)_{\mathrm{DM}}\right] /\left[\left({ }^{176} \mathrm{Lu} /{ }^{177} \mathrm{Hf}\right)_{\mathrm{S}}-\left({ }^{176} \mathrm{Lu} /{ }^{177} \mathrm{Hf}\right)_{\mathrm{DM}}\right]\right\} ;\left({ }^{176} \mathrm{Hf} /{ }^{177} \mathrm{Hf}\right)_{\mathrm{S}}$ and $\left({ }^{176} \mathrm{Lu} /{ }^{177} \mathrm{Hf}\right)_{\mathrm{S}}$ is the value of the sample; $T_{D M 2}=1 / \lambda \times \ln \left\{1+\left[\left({ }^{176} \mathrm{Hf} /{ }^{177} \mathrm{Hf}\right)_{\mathrm{S} . \mathrm{t}}-\left({ }^{176} \mathrm{Hf} /{ }^{177} \mathrm{Hf}\right)_{\mathrm{DM} . \mathrm{t}}\right] /\left[\left({ }^{176} \mathrm{Lu} /{ }^{177} \mathrm{Hf}\right)_{\mathrm{c}}-\left({ }^{176} \mathrm{Lu} /{ }^{177} \mathrm{Hf}\right)_{\mathrm{DM}}\right]\right\}+t ; t$ is the crystallization time of zircon $\left({ }^{176} \mathrm{Hf} /{ }^{177} \mathrm{Hf}\right)_{\mathrm{CHUR} .0}=0.282772,\left({ }^{176} \mathrm{Lu} /{ }^{177} \mathrm{Hf}\right)_{\mathrm{CHUR}}=0.0332,\left({ }^{176} \mathrm{Hf} /{ }^{177} \mathrm{Hf}\right)_{\mathrm{DM}}=0.28325,\left({ }^{176} \mathrm{Lu} /{ }^{177} \mathrm{Hf}\right)_{\mathrm{DM}}=0.0384[45,46] ; \lambda$ $=1.867 \times 10^{-11} / \mathrm{a}\left([47] ;\left({ }^{176} \mathrm{Lu} /{ }^{177} \mathrm{Hf}\right)_{\mathrm{c}}=0.015\right.$.

\subsubsection{Pyrite Re-Os Dating}

Pyrite Re-Os isotope analysis was carried out using a Triton thermal ionization mass spectrometer (TIMS) at the State Key Laboratory of Ore Deposit Geochemistry, Institute of Geochemistry, Chinese Academy of Sciences. $\sim 0.1$ to $0.8 \mathrm{~g}$ sample powder (spiked with ${ }^{185} \mathrm{Re}$ and ${ }^{190} \mathrm{Os}$ ), $2.5 \mathrm{~mL}$ concentrated $\mathrm{HCl}$ and $7.5 \mathrm{~mL}$ concentrated $\mathrm{HNO}_{3}$ were frozen in Carius tubes, which were sealed and heated to $240{ }^{\circ} \mathrm{C}$ for 24 to $48 \mathrm{~h}$. After decomposition and cooling, the Carius tubes were opened and centrifuged and the supernatant was transferred to a $30 \mathrm{~mL}$ PFA flask. Osmium was separated using $4 \mathrm{~mL} \mathrm{CCl}_{4}$ solvent extraction and back extraction following the $\mathrm{HBr}$ method of [48]. The residual solution underwent anion exchange through a column for Re extraction.

Isotope abundances in $\mathrm{OsO}_{3}{ }^{-}$(mass numbers: $233,235,236,237,238,240$ ) was measured using the secondary electron multiplier (SEM) dynamic jumping method. The results were acquired using the stepwise deoxidation method, i.e., using the isothermal model for 
oxygen calibration, and then, the mass fractionation was calibrated using ${ }^{192} \mathrm{Os} /{ }^{188} \mathrm{Os}=$ 3.08271 [49]. Finally, the dilution of the diluent was calculated to determine the contribution of the ${ }^{187} \mathrm{Os} /{ }^{188}$ Os to the sample. Re contents were obtained using an Xseries-2 inductively coupled plasma mass spectrometer. The results were corrected using the Re and Os blanks. Specific dating methods and procedures were described in $[3,50,51]$. The obtained pyrite Re-Os isotopic compositions are tabulated in Table 7.

Table 7. Re-Os isotopic analysis of pyrite in the Shuikoushan deposit.

\begin{tabular}{|c|c|c|c|c|c|c|c|c|}
\hline \multirow{2}{*}{ Sample No. } & \multicolumn{2}{|c|}{$\operatorname{Re}(p p b)$} & \multicolumn{2}{|c|}{ Os (ppb) } & \multicolumn{2}{|c|}{${ }^{187} \mathrm{Os} /{ }^{188} \mathrm{Os}$} & \multicolumn{2}{|c|}{${ }^{187} \mathrm{Re} /{ }^{188} \mathrm{Os}$} \\
\hline & Value & $\begin{array}{c}\text { Uncertainty } \\
(2 \sigma)\end{array}$ & Value & $\begin{array}{c}\text { Uncertainty } \\
(2 \sigma)\end{array}$ & Value & $\begin{array}{c}\text { Uncertainty } \\
(2 \sigma)\end{array}$ & Value & $\begin{array}{c}\text { Uncertainty } \\
(2 \sigma)\end{array}$ \\
\hline ZK2071-1 & 26.898 & 0.562 & 0.0448 & 0.00032 & 105.64 & 0.52 & 42,709 & 943 \\
\hline ZK2071-7 & 11.001 & 0.413 & 0.05748 & 0.0001 & 12.21 & 0.04 & 2375 & 89 \\
\hline ZK2071-8 & 22.23 & 0.145 & 0.04949 & 0.00021 & 33.97 & 0.3 & 11,718 & 91 \\
\hline ZK2071-10 & 13.155 & 0.576 & 0.03216 & 0.00011 & 23.61 & 0.12 & 8009 & 352 \\
\hline ZK2071-11 & 5.632 & 0.125 & 0.03028 & 0.00006 & 9.79 & 0.04 & 2026 & 45 \\
\hline
\end{tabular}

\section{Results}

\subsection{Whole-Rock Major and Trace Element Compositions of the No. 3 Granodiorite}

Rocks have $\mathrm{SiO}_{2}, \mathrm{~K}_{2} \mathrm{O}$, and $\mathrm{Na}_{2} \mathrm{O}$ contents ranging from 55.8 to 62.3 wt. \%, 2.4 to 4.2 wt. \%, and 2.9 to 3.1 wt.\%, respectively (Table 3). On plots of $\mathrm{K}_{2} \mathrm{O}$ versus $\mathrm{SiO}_{2}$ and $\mathrm{A} / \mathrm{CNK}$ versus $\mathrm{A} / \mathrm{NK}$, rocks are plotted mainly in the shoshonite-high-K calc-alkaline field and the metaluminous field, respectively (Figure 7a,b). Rocks have total rare earth element (REE) contents ranging from 200 to $260 \mathrm{ppm}$, and display patterns of enrichment of light REE (LREE, 161 to $215 \mathrm{ppm}$ ) relative to heavy REE (HREE, 36 to $56 \mathrm{ppm}$ ) (Table 3, Figure 8a). In terms of Eu anomalies $\left(\mathrm{Eu} / \mathrm{Eu}^{*}=(\mathrm{Eu})_{\mathrm{N}} /\left[(\mathrm{Sm})_{\mathrm{N}} \times(\mathrm{Gd})_{\mathrm{N}}\right]^{0.5}\right)$, except for one sample that displayed a negative Eu anomaly $\left(\mathrm{Eu} / \mathrm{Eu}^{*}=0.76\right)$, all show no obvious Eu anomalies $\left(\mathrm{Eu} / \mathrm{Eu}^{*}=0.84\right.$ to 0.98$)$ (Figure 8a). In addition, rocks all display pronounced $\mathrm{Nb}$ and $\mathrm{Ta}$ negative anomalies relative to large ion lithophile elements (LILE, e.g., $\mathrm{Rb}$ and $\mathrm{K}$ ) and LREE (e.g., La) (Figure 8b). In general, rocks of the No. 3 granodiorite have similar REE and trace element patterns with other granodiorites in the Shuikoushan deposit (Figure 8a,b), except that the latter show obvious negative $\mathrm{Sr}$ anomalies due to the early fractionation of plagioclase (Figure 8b).
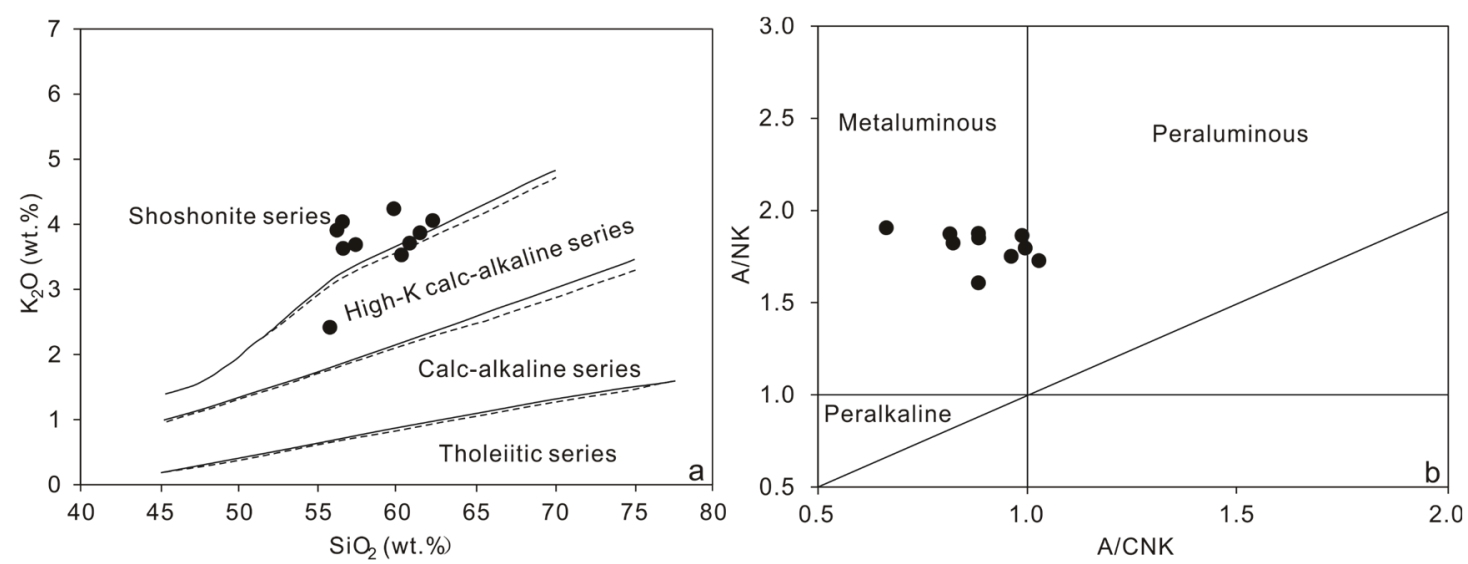

Figure 7. (a) $\mathrm{K}_{2} \mathrm{O}$ versus $\mathrm{SiO}_{2}$ plot for classification of rocks from the No. 3 granodiorite; (b) A/NK versus A/CNK diagram shows that the No. 3 granodiorite has a metaluminous geochemical affinity. 

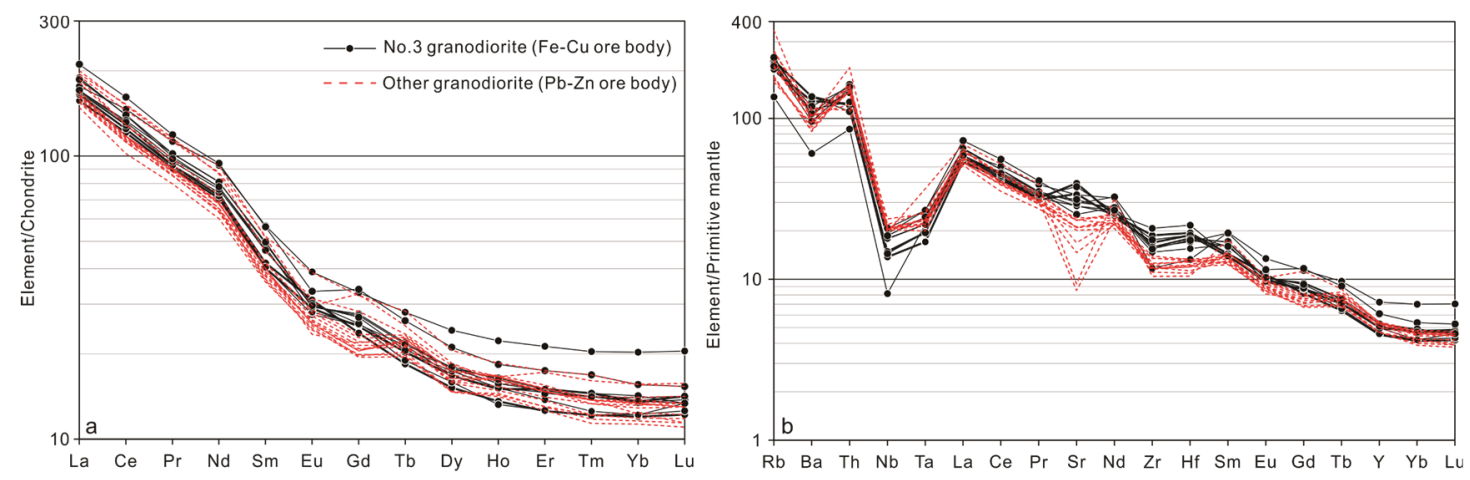

Figure 8. Chondrite-normalized REE (a) and primitive mantle normalized trace element (b) diagrams for rocks of the No. 3 granodiorite and other granodiorites in the Shuikoushan deposit. Data of other granodiorite are from [30], chondrite and primitive mantle values are from [52].

\subsection{Rare Earth Element and Ti Contents of Zircons}

Zircon grains $(n=19)$ have total REE in the range of 373.5 to $1230.6 \mathrm{ppm}(678.6$ ppm on average), with LREE/HREE of 0.02:0.31 on average. The europium anomaly and cerium anomaly $\left(\mathrm{Ce} / \mathrm{Ce}^{*}=(\mathrm{Ce})_{\mathrm{N}} /\left[(\mathrm{La})_{\mathrm{N}} \times(\mathrm{Nd})_{\mathrm{N}}\right]^{0.5}\right)$ are 0.45 to 0.55 and 1.12 to 159.13 , respectively. Zircon grains have Ti contents ranging in the range of 0.2 to $5.5 \mathrm{ppm}$ (Table 4). Based on the Ti-in-zircon thermometer of [53], the zircon crystallization temperature is estimated to be 547 to $782{ }^{\circ} \mathrm{C}$. It is noted that the No. 10 zircon grain likely contains rutile micro-inclusions, as this grain contains anomalously high Ti contents (45.2 ppm) and has crystallization temperature of up to $\sim 90{ }^{\circ} \mathrm{C}$ (Table 4).

\subsection{Zircon $\mathrm{U}-\mathrm{Pb}$ Geochronology}

Zircon grains $(n=19)$ have Th and $\mathrm{U}$ concentrations in the range of 108 to $567 \mathrm{ppm}$ (257 ppm on average), and 195 to $614 \mathrm{ppm}$ (349 ppm on average), respectively. Their Th/U varies from 0.44 to 0.94 ( 0.72 on average) (Table 5). When ignoring the No. 10 zircon that may contain rutile micro-inclusions, they have ${ }^{206} \mathrm{~Pb} /{ }^{238} \mathrm{U}$ ages of ca. 147 to ca. $165 \mathrm{Ma}$. Most of the ages are clustered within the range of ca. 151 to ca. $157 \mathrm{Ma}$ (Figure 9a,b, Table 5). It is noted that three zircon grains (No. 09, 14, and 16) have much older ages (ca. 162 to ca. $165 \mathrm{Ma}$ ) than ages of the rest (ca. 147 to ca.159 Ma) (Figure 9a,b), and the age difference is obviously beyond the $1 \sigma$ uncertainty of the obtained ages (Table 5 ). To better constrain the age of the No. 3 granodiorite, the three relatively old zircons were ignored in case that they are inherited in origin. On the $\mathrm{U}-\mathrm{Pb}$ concordia diagram, data of the rest zircon grains are plotted basically on or close to the concordia line (Figure 9a). The concordia diagram yields a mean ${ }^{206} \mathrm{~Pb} /{ }^{238} \mathrm{U}$ discordant age of $153.7 \pm 0.58 \mathrm{Ma}(1 \sigma$ standard deviation, $M S W D=$ 2.4) $(n=15$, Figure 9a) [54]), consistent with the weighted average age of $154 \pm 3.1 \mathrm{Ma}(1 \sigma$ standard deviation, $M S W D=2.1$ ) (Figure 9b).

\subsection{Zircon Hf Isotopic Compositions}

Nineteen zircon grains were analyzed for Hf isotopes (Table 6), among which, three old zircons may be inherited in origin and are ignored in this section. The zircon ${ }^{176} \mathrm{Lu} /{ }^{177} \mathrm{Hf}$ and ${ }^{176} \mathrm{Hf} /{ }^{177} \mathrm{Hf}$ ranges from 0.001108 to 0.001729 (0.001483 on average), and 0.282406 to 0.282481 ( 0.282446 on average), respectively (Table 6). The zircon $\varepsilon \mathrm{Hf}(\mathrm{t})$ ranges from -9.6 to -7.0 ( -8.3 on average). The two-stage model ages $\left(T_{\mathrm{DM} 2}\right)$ were calculated to be 1.64 to $1.81 \mathrm{Ga}(1.72 \mathrm{Ga}$ on average), similar to the age of the basement rocks in the Shuikoushan ore field [36]. 

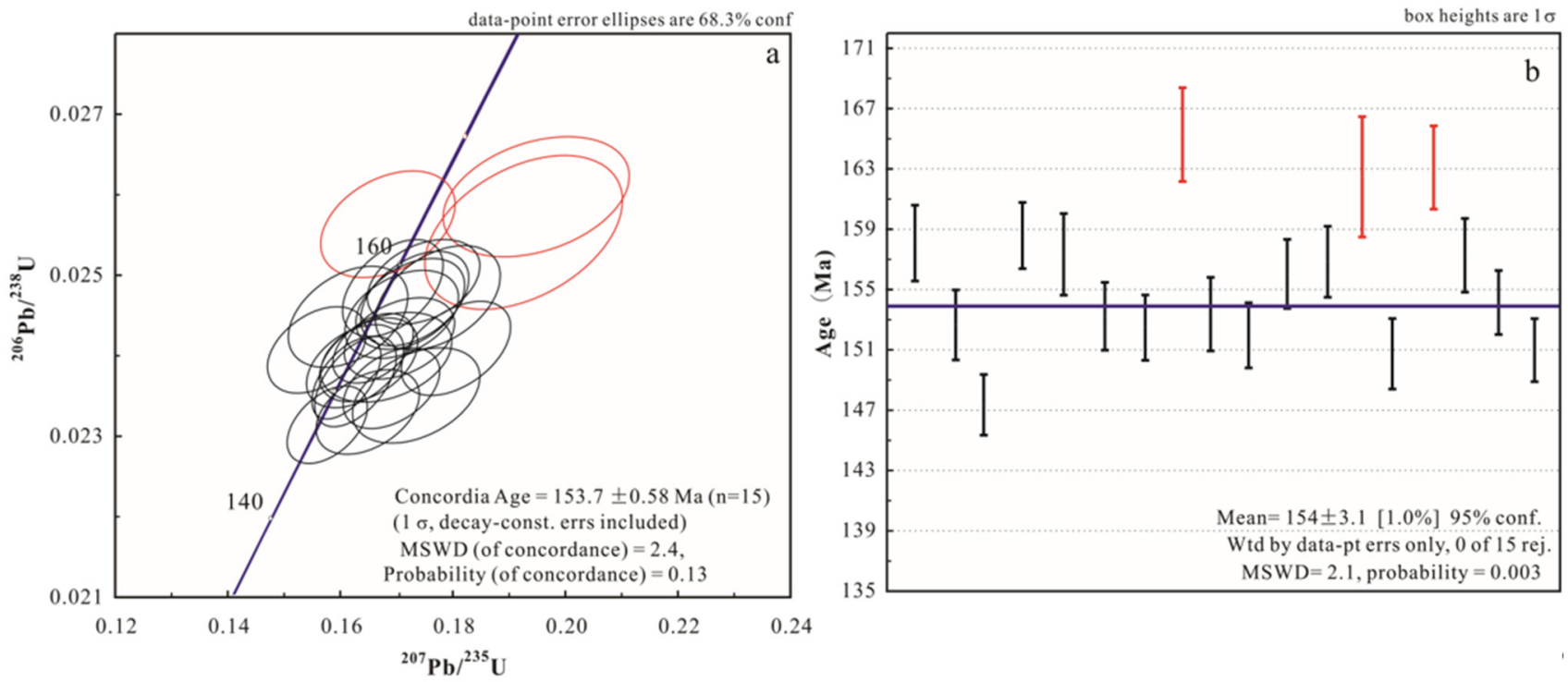

Figure 9. (a) Zircon U-Pb concordia diagram and (b) the weighted average ${ }^{206} \mathrm{~Pb} /{ }^{208} \mathrm{U}$ age of the No. 3 granodiorite. The three relatively older zircons are marked in red colors.

\subsection{Pyrite Re-Os Geochronology}

The average Re and common Os contents of pyrite separates, plus with $2 \sigma$ standard deviation, are in the range of $5.632 \pm 0.125$ to $26.898 \pm 0.562 \mathrm{ppb}$, and $0.03028 \pm 0.00006$ to $0.05748 \pm 0.00010 \mathrm{ppb}$, respectively. The ${ }^{187} \mathrm{Re} /{ }^{188} \mathrm{Os}$ ranges from $2026 \pm 45$ to 11718 \pm 91 ( $2 \sigma$ standard deviation), and ${ }^{187} \mathrm{Os} /{ }^{188} \mathrm{Os}$ ranges from $9.79 \pm 0.04$ to $105.24 \pm 0.52$ ( $2 \sigma$ standard deviation). It is noted that the pyrite separate from ore sample ZK2071-11 is exceptive in that it has much lower Re contents and also lower ${ }^{187} \mathrm{Os} /{ }^{188} \mathrm{Os}$ than the other four pyrite separates (Table 7). This pyrite separate was ignored in order to obtain reliable Re-Os isochron age of Fe-Cu ore mineralization. Using the ISOPLOT software (Model 3, the Berkeley Geochronology Center, Berkeley, CA, USA) [55], a regression analysis was performed, and the intercept on ordinate indicates that the initial ${ }^{187} \mathrm{Os} /{ }^{188} \mathrm{Os}$ ratio of the pyrite is $6.2 \pm 3.1$ ( $2 \sigma$ standard deviation). The obtained isochron age (average value $\pm 2 \sigma$ standard deviation) is $140 \pm 11 \mathrm{Ma}(M S W D=8.1)$ (Figure 10).

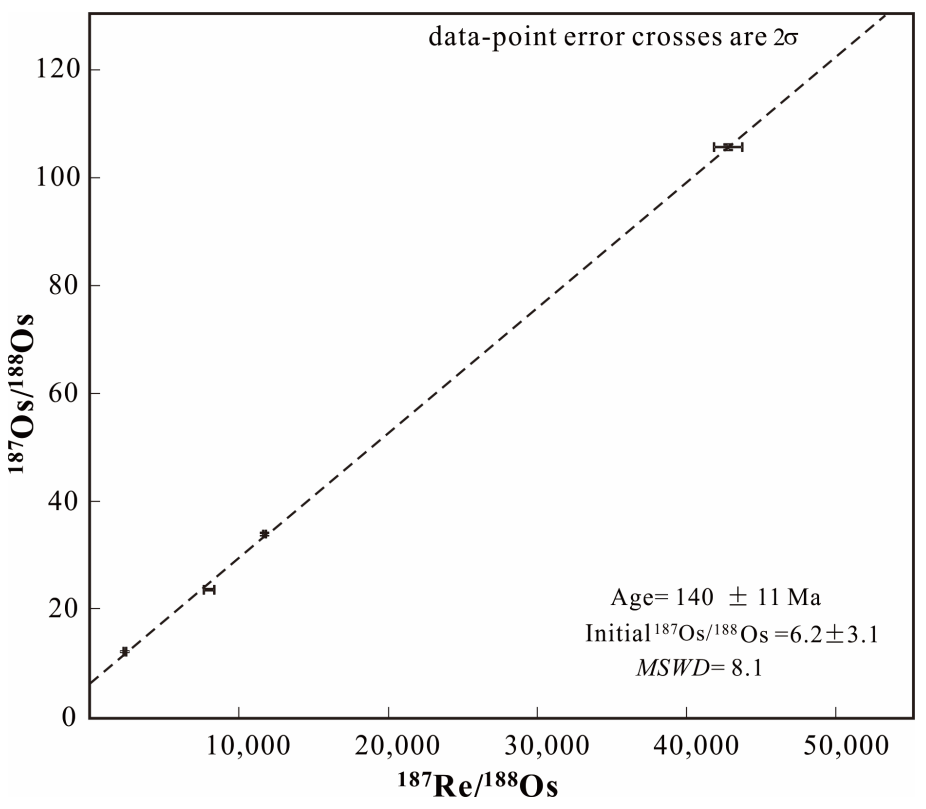

Figure 10. The pyrite Re-Os isochron age of Fe-Cu mineralization in the Shuikoushan deposit. 


\section{Discussion}

\subsection{Emplacement Age and Source of the No. 3 Concealed Granodiorite}

It is known that before using zircon U-Pb ages to constrain the emplacement age of intrusions, it is necessary to confirm that the dated zircon is magmatic in origin so that the obtained ages are meaningful. This is done by considering the following two lines of evidence. Firstly, zircon grains have Th/U (0.44 to 0.94$)$ within the range of magmatic zircons (Th/U > 0.1) [56], and it has been demonstrated that Th/U can be used to distinguish between magmatic, metamorphic, and hydrothermal zircons $[57,58]$. Secondly, zircon grains show strong enrichments in LREE relative to HREE, have negative $\mathrm{Eu}$ anomalies and positive Ce anomalies (Figure 11), and show oscillatory zoning (Figure 6), which are typical of magmatic zircons. Therefore, the obtained zircon U-Pb age (153.7 $\pm 0.58 \mathrm{Ma}$ ) can reliably represent the emplacement age of the No. 3 granodiorite. The obtained age is similar to the reported ages of other granodiorites (163 to $153 \mathrm{Ma}$ ) in the Shuikoushan ore field (Table 1). This, together with their similar REE and trace element patterns (Figure 8), indicate that the No. 3 granodiorite is co-genetic with other granodiorites in the Shuikoushan ore field.

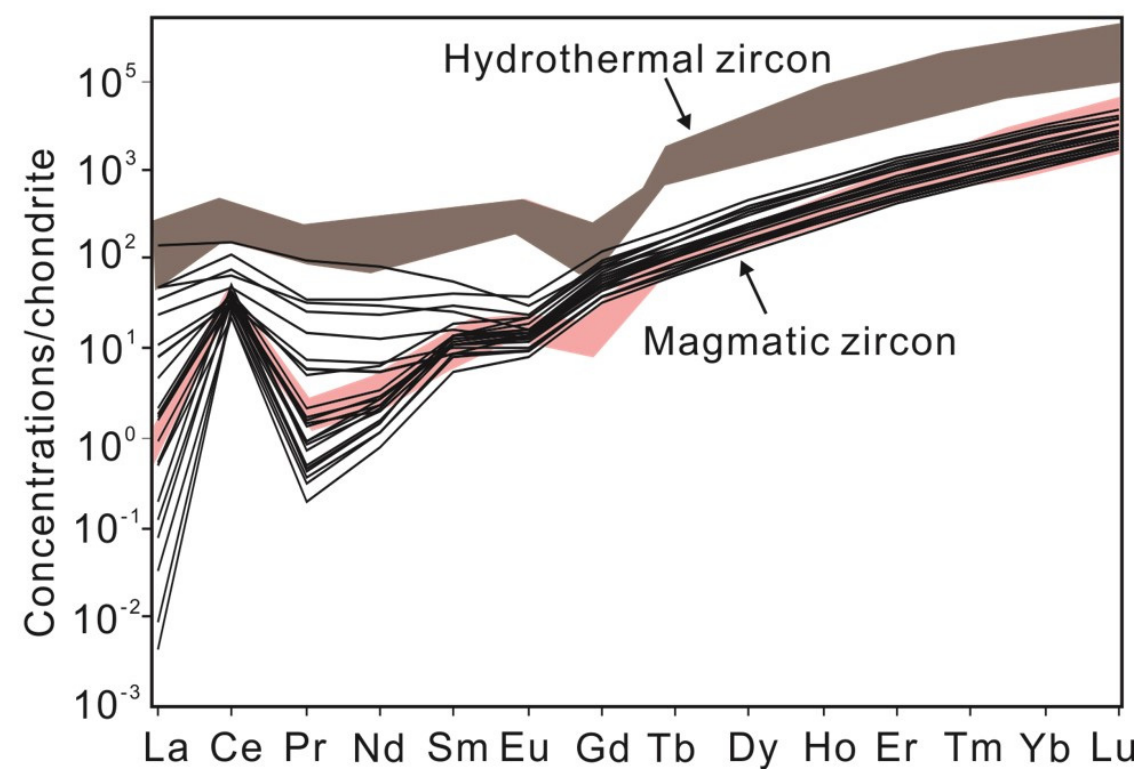

Figure 11. Chondrite normalized rare earth element distribution diagram of zircon grains from the No. 3 granodiorite. Reference fields of hydrothermal and magmatic zircons according to [56], chondrite values according to [52].

The source of the No. 3 granodiorite is evaluated based on in-situ zircon Hf isotopic compositions $[47,59]$. On the diagram of zircon $\varepsilon \mathrm{Hf}(\mathrm{t})$ versus age $(\mathrm{Ma})$, all data points are plotted in or near the field of the lower crust (Figure 12), indicating that materials of this intrusion were likely derived from the lower crust, consistent with the source of other granitoids in the Shuikoushan ore field [2,3]. As the No. 3 granodiorite has zircon two-stage Hf model age ( $\left.T_{2 \mathrm{DM}}\right)$ (1809 to $1644 \mathrm{Ma}$, Table 6 ) similar to the age of the basement rocks in the Shuikoushan ore field [36]), we consider the No. 3 granodiorite, as well as other granitoids in the Shuikoushan ore field were likely derived from partial melting of the Paleoproterozoic basement rocks that are composed mainly by metagreywackes [60]. When looking at a much broader scale, the $\mathrm{W}-\mathrm{Sn}-\mathrm{Pb}-\mathrm{Zn}$ mineralization related granitoids in the Nanling Range are also thought to be derived from partial melting of the lower crustal materials [61], thus the lower crust, particularly the Paleoproterozoic basement metagreywackes may be a common source for granitoids in the whole Nanling Range. 


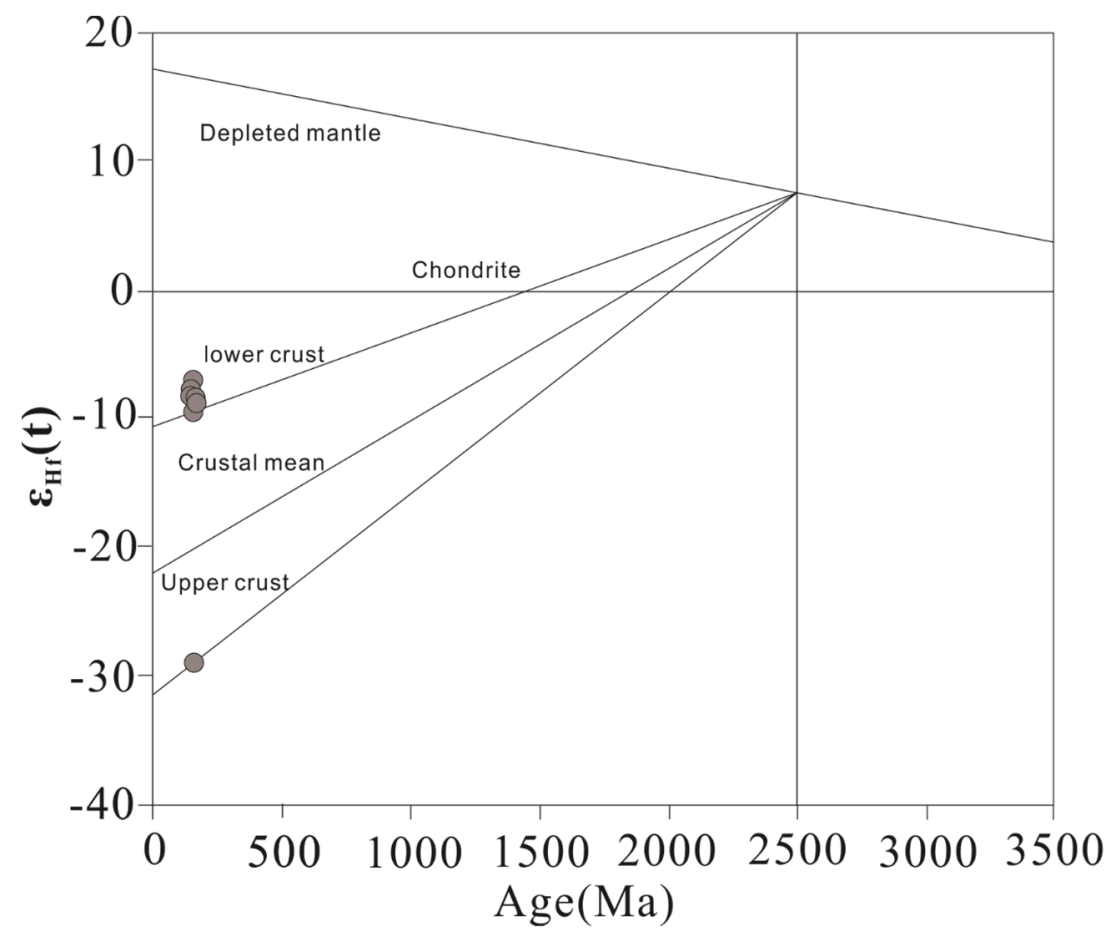

Figure 12. Plot of $\varepsilon \mathrm{Hf}(\mathrm{t})$ vs. U-Pb age of zircon grains from the No. 3 granodiorite.

In summary, our results indicate that the No. 3 granodiorite has the same source and is formed by a common magmatic event with other granodiorites in the Shuikoushan deposit and ore field. As only the No. 3 granodiorite is spatially related to Fe-Cu mineralization, whereas others are often spatially related to $\mathrm{Pb}-\mathrm{Zn}$ mineralization, it does not seem that the No. 3 intrusion itself has a unique 'gene' to form Fe-Cu mineralization in this deposit.

\subsection{Timing and Source of Fe-Cu Mineralization in the Shuikoushan Deposit}

The examined pyrite separates yield consistent isochron ages (Figure 10), suggesting that the Re-Os system of pyrite separates may have remained closed. In addition, homogenization temperatures of fluid inclusions in sulfide minerals in the Shuikoushan ore field vary in the range of 112.0 to $417.7^{\circ} \mathrm{C}$ [9]. These temperature are much lower than the pyrite Re-Os closure temperature $\left(>500^{\circ} \mathrm{C}\right)$ [62], indicating that the Re-Os system of pyrite separates is resistant to any subsequent metamorphism or deformation and can record the timing of crystallization. Therefore, our pyrite Re-Os isochron age provides an important constraint on the timing of Fe-Cu mineralization in the Shuikoushan deposit, which is 140 \pm 11 Ma (Figure 10).

Even considering errors, the timing of Fe-Cu mineralization still post-dates the emplacement age of the No. 3 granodiorite $(153.7 \pm 0.58 \mathrm{Ma})$, and also the timing of $\mathrm{Pb}-\mathrm{Zn}$ mineralization in the deposit (157.8 $\pm 1.4 \mathrm{Ma})$ [60]. Integrating observations such as: (1) the occurrence of Fe-Cu mineralization being controlled by the contact zone of the No. 3 granodiorite with the country rocks, and also by faults [4] (Figure 2b), and (2) that sulfide minerals in this mineralization type mainly occur together with quartz and calcite veinlets that crosscut the magnetite and garnet-diopside skarn (Figures 3 and 4), we consider that the Fe-Cu mineralization in the Shuikoushan deposit was likely produced by post-magmatic hydrothermal fluids after the emplacement of the No. 3 granodiorite. This argument is consistent with the presence of brecciation zones in fold hinge and fault intersections in the vicinity of the ore body, which is evidence of extensive focusing and mixing of hydrothermal fluids [63].

The source of ore-forming materials for $\mathrm{Fe}-\mathrm{Cu}$ mineralization is then evaluated through combining the Re contents, ${ }^{187} \mathrm{Re} /{ }^{188} \mathrm{Os}$, and initial ${ }^{187} \mathrm{Os} /{ }^{188} \mathrm{Os}$ of pyrite separates [21,64-67]. The Re contents (5.6 to $26.9 \mathrm{ppb}$, average at $15.8 \mathrm{ppb})$ and ${ }^{187} \mathrm{Re} /{ }^{188} \mathrm{Os}$ 
(2026 to 42,709, average at 13,367) of pyrite separates are typical of sulfides with low-level highly radiogenic Os (LLHR) (Table 7), similar to Re-Os isotopic features of pyrites from the crust $[67,68]$. The initial ${ }^{187} \mathrm{Os} /{ }^{188}$ Os ratio of pyrite separates $(6.2 \pm 3.1$, Figure 10) is significantly higher than that of the mantle $(0.105$ to 0.152$)[21,69]$, but is close to that of the continental crust (3.63) [70]. These indicate that the ore-forming materials of Fe-Cu mineralization were mainly derived from the crust. To be consistent with this argument, sulfides in ores of the Shuikoushan deposit also have crustal signatures of ${ }^{206} \mathrm{~Pb} /{ }^{204} \mathrm{~Pb}$, ${ }^{207} \mathrm{~Pb} /{ }^{204} \mathrm{~Pb}$, and ${ }^{208} \mathrm{~Pb} /{ }^{204} \mathrm{~Pb}$ that range from 18.4 to $18.5,15.7$ to 15.8 , and 38.6 to 39.1 , respectively [20]. It is therefore likely that Fe-Cu mineralization in the Shuikoushan deposit shares the same material source with the No. 3 granodiorite, which, as mentioned earlier, is related to the Paleoproterozoic basement metagreywackes.

\subsection{A Genetic Model of Fe-Cu Mineralization in the Shuikoushan Deposit}

Based on above discussions, the following conditions need to be considered in order to establish a genetic model for the formation of $\mathrm{Fe}-\mathrm{Cu}$ mineralization in the Shuikoushan deposit. They include: (1) Fe-Cu mineralization is spatially confined within the contact zone between the No. 3 granodiorite and country rocks (limestones of the Qixia and Huitian Formations), where abundant skarns occurred (Figures $2 b$ and 3a), (2) sulfide minerals of this mineralization type crosscut the early formed skarns and magnetite (Figures 3a and 4), (3) this mineralization type formed post the emplacement of the No. 3 granodiorite and is likely related to injection of hydrothermal fluids, and (4) the ore-forming materials of this mineralization type were derived from the Paleoproterozoic basement rocks, similar to the source of the No. 3 granodiorite. By integrating these conditions, a genetic model is proposed, which involves three main events. Firstly, the No. 3 granodiorite intrusion was emplaced into the surrounding country rocks (e.g., limestones), the thermal metamorphism at the contact zone produced magnetite and skarns of the skarn episode (Figures $2 \mathrm{~b}$ and 5). Secondly, post-magmatic hydrothermal fluids transported Fe-Cu sulfide components either from the basement rocks, or through remobilization of these components from the skarns and/or granodiorite. Thirdly, Fe-Cu sulfide minerals, along with quartz were precipitated within the contact zone/faults due to changes in temperature, pressure, etc., and crosscut the early formed skarns and magnetite (Figures $3 a$ and 4). It is noted that the proposed genetic model is just a preliminary one because the source of the post-magmatic hydrothermal fluids cannot be ascertained on the basis of the data in this study. Nevertheless, this study for the first time quantified the timing relationship between Fe- $\mathrm{Cu}$ mineralization and the No. 3 granodiorite in the Shuikoushan deposit, which provides an important basis for understanding the genesis of this mineralization type in the deposit.

\subsection{Implication for the Early Cretaceous Mineralization Event in the Nanling Range}

The timing of $\mathrm{Pb}-\mathrm{Zn}-\mathrm{W}$-Sn mineralization, as well as the emplacement ages of host granoitoids in the Nanling Range have been determined to be 151 to $160 \mathrm{Ma}$ [12-19] (Table 8). The granodiorites (including the No. 3 intrusion) and $\mathrm{Pb}-\mathrm{Zn}$ mineralization in the Shuikoushan deposit have obviously similar ages with $\mathrm{Pb}-\mathrm{Zn}-\mathrm{W}-\mathrm{Sn}$ mineralization and host granoitoids in the Nanling Range (Table 8), indicating that the magmatism and associated $\mathrm{Pb}-\mathrm{Zn}$ mineralization in this deposit can be placed into the metallogenic context of the Nanling Range [9]. The newly discovered Fe-Cu mineralization in the Shuikoushan deposit, however, is exceptive as the timing (140 $\pm 11 \mathrm{Ma})$ is much younger than that of $\mathrm{Pb}-\mathrm{Zn}-\mathrm{W}-\mathrm{Sn}$ mineralization in the Nanling Range. 
Table 8. Ages of some host representative granoitoids and mineralization in the Nanling Range.

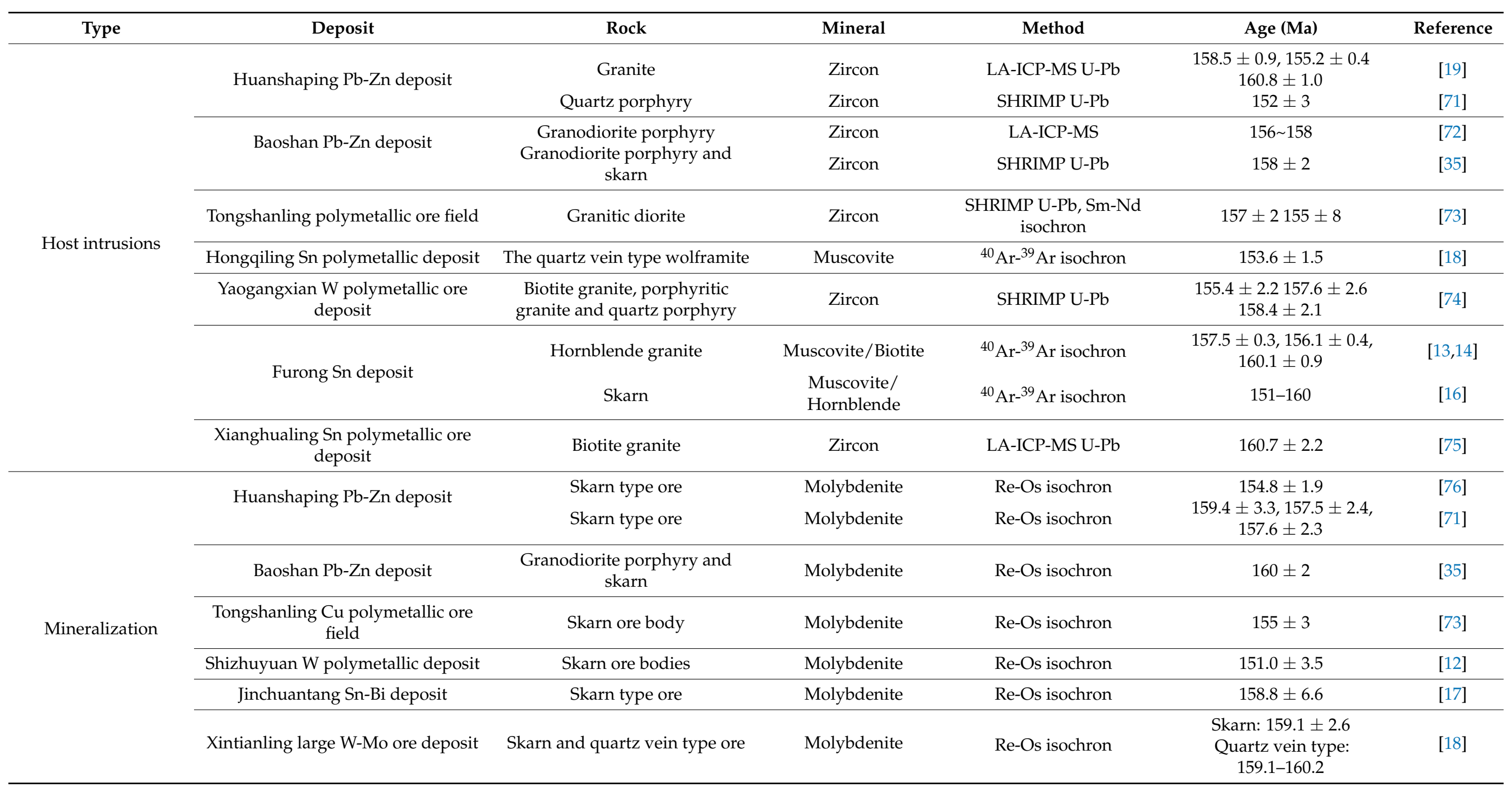


The time interval of 145 to 130 Ma was commonly considered as a period of magmatic quiescence and thus insignificant ore formation in the Nanling Range [15,33,44,77]. However, a recent study by [78] reported an age of 145 to $142 \mathrm{Ma}$ for the muscovite granite in the Dengfuxian pluton in the Nanling Range, and an age of ca. $137 \mathrm{Ma}$ for W-Sn bearing quartz veins that crosscut the Dengfuxian muscovite. Similarly, several other studies also reported 145 to $130 \mathrm{Ma}$ W-Sn mineralization in the North Jiangxi Province and the Southeastern Coastal metallogenic belt that are near the Nanling Range [33,79-82]. Together with the obtained age (140 $\pm 11 \mathrm{Ma})$ of Fe-Cu mineralization in the Shuikoushan deposit, we consider that the mineralization barren interval of 145 to $130 \mathrm{Ma}$ in the Nanling Range may need to be re-visited, and that the Early Cretaceous polymetallic mineralization events are also important and deserve further attentions in the Nanling Range.

\section{Conclusions}

Based on zircon isotopic studies of the No. 3 granodiorite and Re-Os isotopic dating of pyrites collected from Fe-Cu ores in the Shuikoushan deposit, the following conclusions are made:

(1) The No. 3 granodiorite was emplaced at $153.7 \pm 0.58 \mathrm{Ma}$, similar to other granitoids in the Shuikoushan ore field and Nanling Range. Fe-Cu mineralization in the deposit formed at $140 \pm 11 \mathrm{Ma}$, much younger than the No. 3 granodiorite. The younger Fe-Cu mineralization is interpreted as being related to injection of post-magmatic hydrothermal fluids.

(2) The Late Paleoproterozoic basement in the Shuikoushan ore field provides rockforming materials and ore-forming metals of granodiorites and $\mathrm{Fe}-\mathrm{Cu}$ mineralization in this region.

(3) The Early Cretaceous may also be an important mineralization epoch in the Nanling Range.

Author Contributions: Formal analysis, Z.S. and D.X.; supervision, Y.C.; writing-original draft, S.L. All authors have read and agreed to the published version of the manuscript.

Funding: National Natural Science Foundation of China: 41902043 Science and Technology Foundation Project in Guizhou Province: [2019]1316, CAS "Light of West China" Program.

Data Availability Statement: Not Applicable.

Acknowledgments: This study is supported by the National Natural Science Foundation of China (41902043), Science and Technology Foundation Project in Guizhou Province ([2019]1316), CAS “Light of West China" Program. Constructive reviews by the editor and the three anonymous reviewers significantly improved the quality of this paper.

Conflicts of Interest: The authors declare no conflict of interest.

\section{References}

1. Ma, L.Y.; Lu, Y.F.; Mei, Y.P.; Chen, X.Q. Zircon SHRIMP U-Pb dating of granodiorite from Shuikoushan ore-field, Hunan province and its geological significance. Acta Petrol. Sin. 2006, 22, 2475-2482, (In Chinese with English abstract).

2. Zuo, C.H.; Lu, R.; Zhao, Z.X.; Xu, Z.W.; Lu, J.J.; Wang, R.C.; Chen, J.Q. Characterization of element geochemistry, LA-ICP-MS zircon $\mathrm{U}-\mathrm{Pb}$ age, and $\mathrm{Hf}$ isotope of granodiorite in the Shuikoushan deposit, Changning, Hunan Province. Geol. Rev. 2014, 60, 811-823, (In Chinese with English abstract).

3. Li, J.; Zhao, P.P.; Liu, J.G.; Wang, X.C.; Yang Alexandra Wang, G.Q.; Xu, J.F. Reassessment of hydrofluoric acid desilicification in the Carius tube digestion technique for Re-Os isotopic analysis in geological samples. Geostand. Geoanalytical Res. 2015, 39, 17-30. [CrossRef]

4. Chen, P.B.; Liu, J.S.; Wan, K.Y.; Xu, W.J.; Xiong, F.S.; Ren, Z. Geological characteristics and prospecting criteria of skarn iron poly-metallic deposit related to No. 3 granite intrusion in the Shuikoushan ore field, Hunan. Min. Explor. 2016, 7, 450-455, (In Chinese with English abstract).

5. Zeng, M.; Zhang, D.; Zhang, Z.; Li, T.; Li, C.; Wei, C. Structural controls on the Lala iron-copper deposit of the Kangdian metallogenic province, southwestern China: Tectonic and metallogenic implications. Ore Geol. Rev. 2018, 97, 35-54. [CrossRef] 
6. Wan, K.Y.; Chen, P.B.; He, C.X.; Liao, C.H.; Zuo, C.H.; Li, Q.S.; Liu, M.H.; Luo, Q.; Xiang, H.L. The Resource Exploration Report of the Shuikoushan polymetallic Deposit in Hunan Province; Team 217 of Hunan Nonferrous Geological Exploration Bureau Internal Report; Hunan Nonferrous Geological Exploration Bureau: Hunan, China, 2015; p. 120. (In Chinese)

7. Zhen, S.M.; Zhu, X.Y.; Li, Y.S.; Du, Z.Z.; Gong, F.Y.; Gong, X.D.; Qi, F.Y.; Jia, D.L.; Wang, L.L. Zircon U-Pb geochro-nology and Hf isotopic compositions of the monzonite, related to the Xianrenyan Au deposite in Hunan province and its geological significances. J. Jilin Univ. (Earth Sci. Ed.) 2012, 42, 1740-1756, (In Chinese with English abstract).

8. Zhao, Z.X.; Lu, R.; Zuo, C.H.; Miao, B.H.; Xu, Z.W.; Lu, J.J. Discussion on Hunan province Changning Shuikoushan Pb-Zn ore deposit genesis. Acta Mineral. Sin. 2013, s2, 540-541, (In Chinese with English abstract).

9. Huang, J.C.; Peng, J.T.; Yang, J.H.; Zhang, B.L.; Xu, C.X. Precise zircon U-Pb and molybdenite Re-Os dating of the Shuikoushan granodiorite-related $\mathrm{Pb}-\mathrm{Zn}$ mineralization, southern Hunan, south china. Ore Geol. Rev. 2015, 71, 305-317. [CrossRef]

10. Hua, R.M.; Chen, P.R.; Zhang, W.L.; Liu, X.D.; Lu, J.J.; Lin, J.F.; Yao, J.M.; Qi, H.W.; Zhang, Z.S.; Gu, S.Y. Metallogenic systems related to Mesozoic and Cenozoic granitoids in South China. Sci. China Ser. D. 2003, 46, 816-829. [CrossRef]

11. Mao, J.W.; Xie, G.Q.; Guo, C.L.; Yuan, S.D.; Cheng, Y.B.; Chen, Y.C. Spatial-temporal distribution of Mesozoic ore deposits in South China and their metallogenic settings. Geol. J.China Univ. 2008, 14, 510-526, (In Chinese with English abstract).

12. Li, H.Y.; Mao, J.W.; Sun, Y.L.; Zou, X.Q.; He, H.L.; Du, A.D. Re-Os isotopic chronology of the molybdenites in the Shizhuyuan polymetallic tungsten deposit, southern Hunan. Geol. Rev. 1996, 42, 261-271, (In Chinese with English abstract).

13. Mao, J.W.; Stein, H.; Du, A.D.; Zhou, T.F.; Mei, Y.X.; Li, Y.F.; Zang, W.S.; Li, J.W. Molybdenite Re-Os precise dating for molybdenite from $\mathrm{Cu}-\mathrm{Au}-\mathrm{Mo}$ deposits in the middle-lower reaches of Yangtze River belt and its implications for mineralization. Acta Geol. Sin. 2004, 78, 121-131, (In Chinese with English abstract).

14. Mao, J.W.; Xie, G.Q.; Li, X.F.; Zhang, C.Q.; Mei, Y.X. Mesozoic large scale mineralization and multiple lithospheric extension in South China. Earth Sci. Front. 2004, 1, 45-55, (In Chinese with English abstract).

15. Mao, J.W.; Xie, G.Q.; Guo, C.L.; Chen, Y.C. Large-scale W-tin mineralization in the Nanling region, South China: Metallogenic ages and corresponding geodynamic processes. Acta Petrol. Sin. 2007, 23, 2329-2338, (In Chinese with English abstract).

16. Peng, J.T.; Hu, R.Z.; Bi, X.W.; Dai, T.M.; Li, Z.L.; Li, X.M.; Shuang, Y.; Yuan, S.D.; Liu, S.R. 40Ar/39Ar isotopic dating of tin mineralization in Furong deposit of Hunan Province and its geological significance. Miner. Depos. 2007, 3, 237-248, (In Chinese with English abstract).

17. Liu, X.F.; Yuan, S.D.; Wu, S.H. Re-Os dating of the molybdenite from the Jinchuantang tin-bismuth deposit in Hunan Province and its geological significance. Acta Petrol. Sin. 2012, 28, 39-51, (In Chinese with English abstract).

18. Yuan, S.D.; Liu, X.F.; Wang, X.D.; Wu, S.H.; Yuan, Y.B.; Li, X.K.; Wang, T.Z. Geological characteristics and 40Ar-39Ar geochronology of the Hongqiling tin deposit in southern Hunan Province. Acta Petrol. Sin. 2012, 28, 3787-3797, (In Chinese with English abstract).

19. Yuan, Y.B.; Yuan, S.D.; Chen, C.J.; Huo, R. Zircon U-Pb ages and Hf isotopes of the granitoids in the Huangshaping mining area and their geological significance. Acta Petrol. Sin. 2014, 30, 64-78, (In Chinese with English abstract).

20. Lu, R.; Xu, Z.W.; Lu, J.J.; Wang, R.C.; Zuo, C.H.; Zhao, Z.X.; Miao, B.H. Genesis of the Shuikoushan Pb-Zn deposite, Changning City, Hunan province. J. Nanjing Univ. (Nat. Sci.) 2013, 49, 732-746, (In Chinese with English abstract).

21. Walker, R.J.; Carlson, R.W.; Shirey, S.B.; Boyd, F.R. Os, Sr, Nd, and Pb isotope systematics of southern African peridotite xenoliths: Implications for the chemical evolution of subcontinental mantle. Geochim. Cosmochim. Acta 1989, 53, 1583-1595. [CrossRef]

22. Ruiz, J.; Freydier, C.; McCandless, T.; Chesley, J.; Munizaga, F. Re-Os-Isotope systematics of sulfides from base-metal porphyry and manto-type mineralization in Chile. Int. Geol. Rev. 1997, 39, 317-324. [CrossRef]

23. Lambert, D.D.; Foster, J.G.; Frick, L.R. Re-Os isotopic systematics of the Voisey's Bay Ni-Cu-Co magmatic ore system, Labrador, Canada. Lithos 1999, 47, 69-88. [CrossRef]

24. Kirk, J.D.; Ruiz, J.; Kesler, S.E.; Simon, A.; Muntean, J.L. Re-Os age of the pueblo Viejo epithermal deposit, Dominican Republic. Econ. Geol. 2014, 109, 503-512. [CrossRef]

25. Mo, Z.S.; Ye, B.D.; Pan, W.Z.; Wan, S.N. Geology of Granites in Nanling Range; Geological Publishing House: Beijing, China, 1980; pp. 1-363. (In Chinese)

26. Zhou, X.; Sun, T.; Shen, W.; Shu, L.; Niu, A.Y. Petrogenesis of Mesozoic granitoids and volcanic rocks in South China: A response to tectonic evolution. Episodes 2006, 29, 26-33. [CrossRef] [PubMed]

27. Xu, K.Q.; Ding, Y. Viewpoints on the genesis and classification for the tungsten deposits in China. Geol. Rev. 1983, 3, 305-325, (In Chinese with English abstract).

28. Jiang, S.Y.; Zhao, K.D.; Jiang, Y.H.; Ling, H.F.; Ni, P. New type of tin w element and isotope geochemistry. Acta Pet. Sin. 2006, 22, 2509-2516, (In Chinese with English abstract).

29. Jiang, S.Y.; Zhao, K.D.; Jiang, Y.H.; Dai, B.Z. Characteristics and genesis of Mesozoic A type granites and associated mineral deposits in the southern Hunan and northern Guangxi provinces along the Shi-Hang Belt, South China. Geol. J. China Univ. 2008, 14, 496-509, (In Chinese with English abstract).

30. Yang, J.-H.; Peng, J.-T.; Zheng, Y.-F.; Hu, R.-Z.; Bi, X.-W.; Zhao, J.-H.; Huang, J.-C.; Zhang, B.-L. Petrogenesis of the Mesozoic Shuikoushan peraluminous I-type granodioritic intrusion in Hunan Province, South China: Middle-lower crustal reworking in an extensional tectonic setting. J. Asian Earth Sci. 2016, 123, 224-242. [CrossRef]

31. Peng, J.; Zhou, M.-F.; Hu, R.; Shen, N.; Yuan, S.; Bi, X.; Du, A.; Qu, W. Precise molybdenite Re-Os and mica Ar-Ar dating of the Mesozoic Yaogangxian tungsten deposit, central Nanling district, South China. Miner. Deposita 2006, 41, 661-669. [CrossRef] 
32. Peng, J.T.; Hu, R.Z.; Yuan, S.D.; Bi, X.W.; Shen, N.P. The time ranges of granitoid emplacement and related nonferrous metallic mineralization in southern Hunan. Geol. Rev. 2008, 54, 617-625, (In Chinese with English abstract).

33. Mao, J.W.; Cheng, Y.B.; Chen, M.H.; Franco, P. Major types and time-space distribution of Mesozoic ore deposits in South China and their geodynamic settings. Miner. Depos. 2013, 48, 267-294.

34. Li, N.Q.; Peng, C. Shuikoushan Lead-Zinc-Gold-Silver Orefield, Hunan, China; Seismological Publishing House: Beijing, China, 1996; pp. 1-103. (In Chinese)

35. Lu, Y.F.; Ma, L.Y.; Qu, W.J.; Mei, Y.P.; Chen, X.Q. U-Pb and Re-Os isotope geochronology of Baoshan Cu-Mo polymetallic ore deposit in Hunan province. Acta Petrol. Sin. 2006, 22, 2483-2492, (In Chinese with English abstract).

36. Chen, J.F.; Jahn, B.M. Crustal evolution of southeastern China: Nd and Sr isotopic evidence. Tectonophysics 1998, $284,101-133$. [CrossRef]

37. Yu, H.X.; Liu, J.Y. The characteristic and petrogenesis of the felsic-intermediate subvolcanic complex in the Shuikoushan orefield. Geotecton. Et. Metallog. 1997, 1, 32-40, (In Chinese with English abstract).

38. Elhlou, S.; Belousova, E.; Griffin, W.L.; Pearson, N.J.; O’Reilly, S.Y. Trace element and isotopic composition of GJ-red zircon standard by laser ablation. Geochim. Cosmochim. Acta 2006, 70, 158. [CrossRef]

39. Sláma, J.; Košler, J.; Condon, D.J.; Crowley, J.L.; Gerdes, A.; Hanchar, J.M.; Horstwood, M.S.; Morris, G.A.; Nasdala, L.; Norberg, $\mathrm{N}$.; et al. Plešovice zircon-A new natural reference material for $\mathrm{U}-\mathrm{Pb}$ and Hf isotopic microanalysis. Chem. Geol. 2008, 249, 1-35. [CrossRef]

40. Liu, Y.; Gao, S.; Hu, Z.; Gao, C.; Zong, K.; Wang, D. Continental and oceanic crust recycling-induced melt-peridotite interactions in the Trans-North China Orogen: U-Pb dating, Hf isotopes and trace elements in zicons from mantle xenoliths. J. Pet. 2009, 51, 537-571. [CrossRef]

41. Ludwing, K.R. User's Manual for Isoplot 3.75: A Geochronological Toolkit for Microsoft Excel. Berkeley Geochronological Center Special Publication No. 5: Berkeley, CA, USA. 2012. Available online: https://www.geocalculate.com/wp-content/uploads/20 19/10/Isoplot3_75-4_15manual.pdf (accessed on 30 January 2012).

42. Wu, F.-Y.; Yang, Y.-H.; Xie, L.-W.; Yang, J.-H.; Xu, P. Hf isotopic compositions of the standard zircons and baddeleyites used in U-Pb geochronology. Chem. Geol. 2006, 234, 105-126. [CrossRef]

43. Yuan, H.-L.; Gao, S.; Dai, M.-N.; Zong, C.-L.; Günther, D.; Fontaine, G.H.; Liu, X.-M.; Diwu, C. Simultaneous determinations of $\mathrm{U}-\mathrm{Pb}$ age, $\mathrm{Hf}$ isotopes and trace element compositions of zircon by excimer laser-ablation quadrupole and multiple-collector ICP-MS. Chem. Geol. 2008, 247, 100-118. [CrossRef]

44. Li, Q.L.; Li, X.H.; Liu, Y.; Tang, G.Q.; Yang, J.H.; Zhu, W.G. Precise U-Pb and Pb-Pb dating of Phanerozoic baddeleyite by SIMS with oxygen flooding technique. J. Anal. At. Spectrom. 2010, 25, 1107-1113. [CrossRef]

45. Wu, F.Y.; Li, X.H.; Zheng, Y.F.; Gao, S. Lu-Hf isotopic systematics and their applications in petrology. Acta Petrol. Sin. 2007, 23, 185-220, (In Chinese with English abstract).

46. Rubatto, D.; Gebauer, D. Use of cathodoluminescence for U-Pb zircon dating by ion microprobe: Some examples from the Western Alps. In Cathodoluminescence in Geosciences; Pagel, M., Barbin, V., Blanc, P., Ohnenstetter, D., Eds.; Springer: Berlin, Germany, 2000; pp. 373-400.

47. Griffin, W.L.; Pearson, N.J.; Belousova, E.; Jackson, S.E.; van Achterbergh, E.; O’Reilly, S.Y.; Shee, S.R. The Hf isotope composition of cratonic mantle: LA-MC-ICPMS analysis of zircon megacrysts in kimberlites. Geochim. Cosmochim. Acta 2000, 64, $133-147$. [CrossRef]

48. Cohen, A.S.; Waters, F.G. Separation of osmium from geological materials by solvent extraction for analysis by thermal ionisation mass spectrometry. Anal. Chim. Acta 1996, 332, 269-275. [CrossRef]

49. Nier, A.O. The Isotopic Constitution of Osmium. Phys. Rev. 1937, 52, 885. [CrossRef]

50. Li, J.; Xu, J.F.; Suzuki Katsuhiko He, B.; Xu, Y.G.; Ren, Z.Y. Os, Nd and Sr isotope and trace element geochemistry of the Muli picrites: Insights into the mantle source of the Emeishan Large Igneous Province. Lithos 2010, 119, 108-122. [CrossRef]

51. Li, J.; Jiang, X.Y.; Xu, J.F.; Zhong, L.F.; Wang, X.C.; Wang, G.Q.; Zhao, P.P. Determination of platinum-group elements and Re-Os isotopes using ID-ICP-MS and N-TIMS from a single digestion after two-stage column separation. Geostand. Geoanalytical Res. 2014, 38, 37-50. [CrossRef]

52. Sun, S.-S.; McDonough, W.F. Chemical and isotopic systematics of oceanic basalts: Implications for mantle composition and processes. Geol. Soc. London. Spéc. Publ. 1989, 42, 313-345. [CrossRef]

53. Watson, E.B.; Wark, D.A.; Thomas, J.B. Crystallization thermometers for zircon and rutile. Contrib. Miner. Pet. 2006, 151, 413-433. [CrossRef]

54. Micheletti, F.; Fornelli, A.; Piccarreta, G.; Barbey, P.; Tiepolo, M. The basement of Calabria (southern Italy) within the context of the Southern European Variscides: LA-ICPMS and SIMS U-Pb zircon study. Lithos 2008, 104, 1-11. [CrossRef]

55. Ludwig, K.R. Isoplot/Ex: A geochronological toolkit for Microsoft Excel; Berkeley Geochronology Center Special Publication: Version 3.0; Berkeley Geochronology Center: Berkeley, CA, USA, 1999; p. 49. Available online: https://searchworks.stanford.edu/view/ 6739593 (accessed on 27 August 2003).

56. Hoskin, P.O. Trace-element composition of hydrothermal zircon and the alteration of Hadean zircon from the Jack Hills, Australia. Geochim. Cosmochim. Acta 2005, 69, 637-648. [CrossRef]

57. Belousova, E.A.; Griffin, W.L.; O' Reilly, S.Y.; Fisher, N.L. Igneous zircon: Trace element composition as an indicator of source rock type. Contrib. Miner. Pet. 2002, 143, 602-622. [CrossRef] 
58. Hermann, J.; Rubatto, D.; Korsakov, A.; Shatsky, V.S. Multiple zircon growth during fast exhumation of diamondiferous, deeply subducted continental crust (Kokchetav Massif, Kazakhstan). Contrib. Miner. Pet. 2001, 141, 66-82. [CrossRef]

59. Bouvier, A.; Vervoort, J.D.; Patchett, P.J. The Lu-Hf and Sm-Nd isotopic composition of CHUR: Constraints from unequilibrated chondrites and implications for the bulk composition of terrestrial planets. Earth Planet. Sci. Lett. 2008, 273, 48-57. [CrossRef]

60. Huang, J.C. Geochronology and Petrogenetic-Metallogenic Mechanisms in the Shuikoushan Pb-Zn-Au Deposit; University of Chinese Academy of Sciences: Hunan, China, 2016; (In Chinese with English abstract).

61. Mao, J.W.; Pirajno, F.; Xiang, J.F.; Gao, J.J.; Ye, H.S.; Li, Y.F.; Guo, B.J. Mesozoic molybdenum deposits in the east Qinling-Dabie orogenic belt: Characteristics and tectonic settings. Ore Geol. Rev. 2011, 43, 264-293. [CrossRef]

62. Li, S.D.; Wang, Z.G.; Wang, K.Y.; Cai, W.Y.; Peng, D.W.; Xiao, L.; Li, J. Re-Os Pyrite geochronological evidence of three mineralization styles within the Jinchang Gold Deposit, Yanji-Dongning Metallogenic Belt, Northeast China. Minerals 2018, 8, 448. [CrossRef]

63. Zhang, Y.; Lin, G.; Roberts, P.; Ord, A. Numerical modelling of deformation and fluid flow in the Shuikoushan district, Hunan Province, South China. Ore Geol. Rev. 2007, 31, 261-278. [CrossRef]

64. Walker, R.; Morgan, J.; Horan, M.; Czamanske, G.; Krogstad, E.; Fedorenko, V.; Kunilov, V. Re-Os isotopic evidence for an enriched-mantle source for the Noril'sk-type, ore-bearing intrusions, Siberia. Geochim. Cosmochim. Acta 1994, 58, $4179-4197$. [CrossRef]

65. Foster, J.G.; Lambert, D.D.; Frick, L.R.; Maas, R. Re-Os isotopic evidence for genesis of Archaean nickel ores from uncontaminated komatiites. Nature 1996, 382, 703-706. [CrossRef]

66. Mao, J.W.; Wang, Z.L. A preliminary study on time limits and geodynamic setting of large-scale metallogeny in east China. Miner. Depos. 2000, 9, 289-299, (In Chinese with English abstract).

67. Stein, H.J.; Morgan, J.W.; Schersten, A. Re-Os Dating of Low-Level Highly Radiogenic (LLHR) Sulfides:The Harnas Gold Deposit, Southwest Sweden, Records Continental-Scale TectonicEvents. Econ. Geol. 2000, 95, 1657-1671. [CrossRef]

68. Arne, D.; Bierlein, F.P.; Morgan, J.W.; Stein, H.J. Re-Os dating of sulfides associated with gold mineralization in central Victoria, Australia. Econ. Geol. 2001, 96, 1455-1459. [CrossRef]

69. Shirey, S.B.; Walker, R.J. The Re-Os isotope system in cosmochemistry and high-temperature geochemistry. Annu. Rev. Earth Planet. Sci. 1998, 26, 423-500. [CrossRef]

70. Palmer, M.R.; Turekian, K.K. 187Os/186Os in marine manganese nodules and the constraints on the crustal geochemistries of rhenium and osmium. Nat. Cell Biol. 1986, 319, 216-220. [CrossRef]

71. Lei, Z.H.; Chen, F.W.; Chen, Z.H.; Xu, Y.M.; Gong, S.Q.; Li, H.Q.; Mei, Y.P.; Qu, W.J.; Wang, D.H. Petrogenetic and metallogenic age determination of the Huangshaping $\mathrm{Pb}-\mathrm{Zn}$ polymetallic deposit and its geological significance. Acta Geosci. Sin. 2010, 31, 532-540, (In Chinese with English abstract).

72. Xie, Y.C.; Lu, J.J.; Ma, D.S.; Zhang, R.Q.; Gao, J.F.; Yao, Y. Origin of granodiorite porphyry and mafic microgranular enclave in the Baoshan $\mathrm{Pb}-\mathrm{Zn}$ polymetallic deposit, southern Hunan Province: Zircon U-Pb chronological, geochemical and Sr-Nd-Hf isotopic constraints. Acta Petrol. Sin. 2013, 29, 4186-4214.

73. Lu, Y.Y.; Fu, J.M.; Cheng, S.B.; Liu, S.S.; Li, C.B.; Zhang, L.G.; Ma, L.Y. Rock-Forming and Ore-Forming Ages of Tongshanling $\mathrm{Cu}$ Polymetallic Ore-Field in Southern Hunan Province. Geotecton. Et. Metallog. 2015, 39, 1061-1071, (In Chinese with English abstract).

74. Li, S.T.; Wang, J.B.; Zhu, X.Y.; Li, C. Re-Os dating of molybdenite and S isotope analysis of the Yaogangxian W polymetallic deposits in Hunan province and their geological significance. Geoscience 2011, 25, 228-235, (In Chinese with English abstract).

75. Xuan, Y.S.; Yuan, S.D.; Yuan, Y.B.; Mi, J.R. Zircon U-Pb age, geochemistry and Petrogenesis of Jianfengling pluton in southern Hunan Province. Miner. Depos. 2014, 33, 1379-1390.

76. Yao, J.; Hua, R.; Qu, W.; Qi, H.; Lin, J.; Du, A. Re-Os isotope dating of molybdenites in the Huangshaping Pb-Zn-W-Mo polymetallic deposit, Hunan Province, South China and its geological significance. Sci. China Ser. D Earth Sci. 2007, 50, 519-526. [CrossRef]

77. Hua, R.M.; Li, G.L.; Zhang, W.L.; Hu, D.Q.; Chen, P.R.; Chen, W.F.; Wang, X.D. A tentative discussion on differences between large-scale W and tin mineralizations in South China. Min. Depos. 2010, 29, 9-23, (In Chinese with English abstract).

78. Xiong, Y.-Q.; Shao, Y.-J.; Cheng, Y.; Jiang, S.-Y. Discrete Jurassic and Cretaceous Mineralization Events at the Xiangdong W(-Sn) Deposit, Nanling Range, South China. Econ. Geol. 2020, 115, 385-413. [CrossRef]

79. Huang, L.; Jiang, S. Highly fractionated S-type granites from the giant Dahutang tungsten deposit in Jiangnan orogen, southeast China: Geochronology, Petrogenesis and their relationship with W-mineralization. Lithos 2014, 202, 207-226. [CrossRef]

80. Pan, X.; Hou, Z.; Li, Y.; Chen, G.; Zhao, M.; Zhang, T.; Zhang, C.; Wei, J.; Kang, C. Dating the giant Zhuxi W-Cu deposit (Taqian-Fuchun Ore Belt) in South China using molybdenite Re-Os and muscovite Ar-Ar system. Ore Geol. Rev. 2017, 86, 719-733. [CrossRef]

81. Liu, P.; Mao, J.; Pirajno, F.; Jia, L.; Zhang, F.; Li, Y. Ore genesis and geodynamic setting of the Lianhuashan porphyry tungsten deposit, eastern Guangdong Province, SE China: Constraints from muscovite 40Ar-39Ar and zircon U-Pb dating and Hf isotopes. Miner. Depos. 2017, 5, 1-18. [CrossRef]

82. Liu, P.; Mao, J.; Santosh, M.; Xu, L.; Zhang, R.; Jia, L. The Xiling Sn deposit, eastern Guangdong Province, southeast China: A new genetic model from 40Ar/39Ar muscovite and U-Pb cassiterite and zircon geochronology. Econ. Geol. 2018, 113, 511-530. [CrossRef] 